\title{
Ein Botaniker in der Papiergeschichte: Offene und geschlossene Kooperationen in den Wissenschaften um 1900
}

\author{
Josephine Musil-Gutsch und Kärin Nickelsen
}

A Botanist in the History of Paper: Open and Closed Cooperations in the Sciences Around 1900

\begin{abstract}
The paper uses the example of historical paper research in Vienna around 1900 in order to analyze the dynamics of scientific cooperation between the natural sciences and the humanities. It focuses on the Vienna-based plant physiologist Julius Wiesner (1838-1916), who from 1884 to 1911 studied medieval paper manuscripts under the microscope in productive cooperation with paleographers, archaeologists and orientalists (Josef Karabacek, Marc Aurel Stein, Rudolf Hoernle). The paper examines why these cooperations succeeded and how they developed over time. Here we distinguish between two forms of cooperation: while Wiesner initially worked only reactively, in a "closed cooperation", he later entered into "open cooperations", in which both parties defined research questions and methods. This form of cooperation proved particularly successful, but at the same time was especially demanding because, in addition to contributing one's own skills, it required considerable "interlocking expertise" (Andersen 2016). This was favored because the historical auxiliary sciences were in a phase of upheaval. Wiesner contributed his knowledge of technical microscopy and developed a genuine interest in historical questions, while the humanists were prepared to open themselves to scientific processes and ultimately acknowledged Wiesner as a historian of paper in his own right.
\end{abstract}

Keywords: The Two Cultures, History of sciences and humanities, History of auxiliary sciences, History of botany, Interdisciplinary cooperation, Expertise, Fin-de-siècle Vienna

Die Studie analysiert die Dynamik wissenschaftlicher Kooperation zwischen Natur- und Geisteswissenschaften an einem Beispiel aus der historischen Papierforschung in Wien um 1900. Im Mittelpunkt steht der Wiener Pflanzenphysiologe Julius Wiesner (1838-1916), der ab 1884 (und bis 1911) mittelalterliche Papiermanuskripte unter dem Mikroskop prüfte. Dies erfolgte in produktiver Zusammenarbeit mit Paläographen, Archäologen und Orientalisten (Josef Karabacek, Marc Aurel Stein, Rudolf Hoernle). Der Aufsatz untersucht, warum dies gelang und wie die Zusammenarbeit sich entwickelte. Wir unterscheiden dabei zwei Formen der Kooperation: Während Wiesner anfangs nur reaktiv zuarbeitete, in einer "geschlossenen Kooperation", trat er später in "offene Kooperationen" ein, in der beide Parteien die Fragen definieren und geeignete Methoden entwickeln. Diese Form der Kooperation erwies sich als besonders erfolgreich - zugleich war sie besonders anspruchsvoll, denn neben fachlicher Expertise erforderte sie erhebliche "Integrationsexpertise" (Andersen 2016). Dies wurde begünstigt durch die lokale Konstellation in einer Umbruchphase der historischen Hilfswissenschaften. Wiesner konnte seine Kenntnisse der technischen Mikroskopie einbringen und gewann ein genuines Interesse an den historischen Fragen, während die Geisteswissenschaftler bereit waren, sich naturwissenschaftlichen Verfahren gegenüber zu öffnen und Wiesner letztlich als Papierhistoriker eigenen Rechts anzuerkennen.

Schlüsselwörter: Zwei Kulturen, Geschichte der Geisteswissenschaften, Geschichte der Hilfswissenschaften, Geschichte der Botanik, Interdisziplinäre Kooperation, Expertise, Wien um 1900 


\section{Einleitung}

„Bei all dem gebe ich wohl gerne zu dass ich das Vorurtheil habe, dass es mit Ihrer Ansicht über die Chinesen seine Richtigkeit haben könnte. Nur bin ich noch nicht recht überzeugt dass dieser Punkt historisch schon entschieden ist. ${ }^{\text {“1 }}$ Mit diesen Worten beendete der deutsch-britische Indologe Rudolf Hoernle am 1. Januar 1912 einen Brief an den Wiener Pflanzenphysiologen Julius Wiesner. Hoernles Kritik bezog sich auf die jüngste papierhistorische Publikation von Wiesner zu den Ursprüngen des Hadernpapiers, das im Mittelalter als Schreibstoff gebräuchlich war (Wiesner 1911). Wiesner argumentierte darin, dass das Hadernpapier ursprünglich aus China kam und nicht, wie bisher angenommen wurde, aus den arabischen Ländern. Hoernle war zwar geneigt Wiesners These zuzustimmen, hielt diese aber für noch nicht ausreichend belegt.

Wie kam es dazu, dass ein Philologe mit einem Botaniker über den Ursprung bestimmter Papiersorten diskutierte? Im Jahre 1884 hatte sich Wiesner zum ersten Mal mit altem Papier beschäftigt, als der Wiener Orientalist Josef Karabacek ihn um die mikroskopische Analyse der Papierfasern alter arabischer Schriftstücke bat. Als Pflanzenphysiologe, Warenkundler und Mikroskopiker war Wiesner für diese Aufgabe prädestiniert. Bald schon entwickelte er ein erhebliches Eigeninteresse an dem Gebiet und erweiterte und verfeinerte seine Methoden der Papieranalyse. Über die nächsten etwa dreißig Jahre folgten weitere Kooperationen, in denen Wiesner sich allmählich von der bloßen Zuarbeit emanzipierte und selbstständig für historische Thesen eintrat. Durch seine außergewöhnliche Kompetenz in der mikroskopischen und mikrochemischen Papieranalyse stieg Wiesner schließlich zur international anerkannten Autorität in der historischen Papierforschung auf.

Es waren vor allem drei Personen, mit denen Wiesner zusammenarbeitete: von 1884 bis 1888 mit Karabacek, von 1900 bis 1903 mit Hoernle und in den Jahren 1902 bis 1904 und 1910 bis 1911 mit dem deutschungarischen Archäologen Aurel Stein. Die Dynamik und Form der Kooperationen ist in reicher Korrespondenz dokumentiert; zudem entstanden breit rezipierte Publikationen von Wiesner zur Papiergeschichte (Wiesner 1887, 1902, 1904b, 1911). Es handelt sich dabei um frühe und besonders interessante Beispiele für die erfolgreiche Zusammenarbeit von Wissenschaftlern über die Grenze der so genannten „Zwei Kulturen“ hinweg. Seit der Physiker und Schriftsteller C. P. Snow diesen Begriff 1959 prägte (Gloy 2002; Jost \& Rohbeck 2007; Jardine 2010; Welsh 2008), wurde seine These einer Kluft zwischen Geistes- und Naturwissenschaften von der Wissenschaftsgeschichte vielfach diskutiert (u.a. Bod \& Kursell 2015: 337-340; Krämer 2018: 5-14). Einigkeit besteht inzwischen darüber, dass diese Kul- 
turen sich vergleichsweise spät ausprägten; ein übliches Narrativ verortet diesen Prozess in der zweiten Hälfte des 19. Jahrhunderts, mit der Teilung philosophischer Fakultäten als Ausdruck vollzogener Demarkation (Krämer 2018; Hamann 2018; Bouterse \& Karstens 2015). Nur selten fiel dabei der Blick auf Grenzgänge, Transferprozesse und Zusammenarbeit, wie sie aus dem hier betrachteten Beispiel hervorgeht.

Die Kooperation, zum Teil auch Konkurrenz, von Biologie und Geschichtswissenschaften bei der Erklärung von Sachverhalten der Vergangenheit wurde jüngst zunehmend zum Gegenstand historischer, aber auch gegenwartsbezogener Wissenschaftsreflexion (Bösl 2017; Jones 2018; o.A. 2014). ${ }^{2}$ Unser Beispiel zeigt, dass diese Zusammenarbeit kein neues Phänomen ist, sondern spätestens im 19. Jahrhundert begann. Im hier untersuchten Fall waren es Historiker, die die Kooperation mit einem Botaniker suchten. Vor dem Hintergrund des damaligen Prestigegefälles der Disziplinen mag dies überraschen, da sich die „historischen Wissenschaften [...] im späteren 19. Jh. auf der Höhe ihres Ansehens" befanden (Mommsen 1997: 32), während sich die noch junge Disziplin der Pflanzenphysiologie gerade erst zu legitimieren versuchte (Wiesner 1910). ${ }^{3}$ Wiesner war einer der namhaftesten Vertreter dieses neuen Feldes (Gabriel 2007: 55-56; Babler 1935: 463); in diesem Aufsatz geht es darum, wie er zudem zum europaweit gefragten Experten für altes Papier aufstieg. Wie kam es zur Zusammenarbeit von Wiesner mit Paläographen und Orientalisten um 1900? Und welche Bedingungen begünstigten den erfolgreichen Verlauf dieser Kooperation?

$\mathrm{Zu}$ drei Bereichen möchte dieser Aufsatz einen Diskussionsbeitrag leisten: erstens zur Geschichte der historischen Papierforschung; zweitens zu Varianten kooperativer Forschung und ihrer Dynamik; drittens zu den besonderen Anforderungen an interdisziplinäre Kooperationen, insbesondere über die Grenze der Wissenschaftskulturen hinweg. Im Folgenden etablieren wir zunächst unser begriffliches Inventar zur Beschreibung interdisziplinärer Kooperationen, insbesondere werden wir verschiedene Arten von Expertise sowie zwei Typen wissenschaftlicher Kooperation einführen. Vor diesem Hintergrund werden wir dann untersuchen, auf welche Weise und zu welchen Fragen Wiesner mit Karabacek, Hoernle und Stein zusammenarbeitete. Dabei wird sich zeigen, dass auch der besondere lokale Kontext von Bedeutung war, die Wissenschaftslandschaft in Wien gegen Ende des 19. Jahrhunderts. Wir schließen mit einigen Überlegungen zur Kooperation über die Grenzen von Disziplinen und Wissenschaftskulturen hinweg. 


\section{Kooperation zwischen Disziplinen}

Kooperation verstehen wir in diesem Aufsatz als „das Zusammenwirken von zwei oder mehreren Akteuren [...], in Form bewusst gewählter Handlungen“, deren Ziele positiv interdependent verknüpft sind; das heißt beide Parteien können ihre Ziele nur dann erreichen, wenn auch die je andere Partei die ihren erreicht (Nickelsen 2014: 356; Nickelsen \& Schürch 2020). ${ }^{4}$ Oft geht es bei diesem Zusammenwirken darum, Ziele zu erreichen, die alleine nicht oder nur ungleich mühsamer erreicht werden können. So lassen sich umfangreiche und zeitaufwändige Projekte besser (oder überhaupt nur) bewältigen, indem man die Arbeit auf viele Hände und Köpfe verteilt, etwa die naturhistorische Beschreibung der Welt oder die Sequenzierung des menschlichen Genoms (Daston 2007; Daston \& Park 2002; Gannett 2019; Parker et al. 2013). Doch gibt es auch kleinräumige Forschungsfragen, die nur bearbeitet werden können, indem man verschiedene Expertisen kombiniert, das heißt verschiedene Wissensbestände und methodische Kompetenzen, auch über Disziplinengrenzen hinweg (Andersen 2016; Andersen \& Wagenknecht 2013; Wray 2002). Um diese Form der Zusammenarbeit geht es uns im Folgenden. Disziplinen verstehen wir dabei konservativ und deflationär im Sinne intellektuell und institutionell abgegrenzter akademischer Fächer, so wie sie um 1900 vorlagen.

In interdisziplinären Kooperationen wirken Wissenschaftler mit unterschiedlichem disziplinärem Hintergrund und fachlicher Expertise zusammen, um neue Forschungsergebnisse zu produzieren (Andersen \& Wagenknecht 2013: 1881; Nickelsen \& Schürch 2020). Mindestens einer der Kooperationspartner verfügt dabei über Kompetenzen und Wissensbestände, über die der andere nicht verfügt, diese aber benötigt, um die eigenen Ziele zu erreichen. ${ }^{5}$ Hanne Andersen bezeichnet dies in ihrer Analyse als contributory expertise: die inhaltliche Expertise, mit der zum gemeinsamen Projekt beigetragen wird (Andersen 2016: 2-3). ${ }^{6}$ Um die Kooperation zum Erfolg zu bringen, muss diese ergänzt werden durch interlocking expertise, im Folgenden (etwas holperig) übersetzt als „Integrationsexpertise“, die es ermöglicht, dass die unterschiedlichen inhaltlichen Expertisen mit Blick auf das Erkenntnisziel produktiv ineinander greifen (Andersen 2016: 3). In der Regel muss dazu mindestens eine Person mit den Fragestellungen, Methoden und Kategorien der anderen Disziplin soweit vertraut sein, dass sie diese mit eigenen Beiträgen und Fertigkeiten verknüpfen kann. Im besten Fall sind beide Partner in diesem Sinne kompetent (Andersen 2016: 4). Je unterschiedlicher die Disziplinen sind, und je enger die Befunde integriert werden sollen, desto höher ist der Anspruch an diese zweite Form der Expertise (Andersen 2016: 6). ${ }^{7}$ Je deutlicher die Integrationsexpertise 
ausgeprägt ist, kann man anders herum schließen, desto fruchtbarer und erfolgsversprechender ist die interdisziplinäre Zusammenarbeit.

Die meisten wissenschaftshistorischen Studien zur Dynamik interdisziplinärer Kooperation beziehen sich auf Beispiele des 20. und 21. Jahrhunderts; zudem richten sie den Blick ganz überwiegend auf kooperative Großprojekte (z. B. Galison 2003; Parker et al. 2013). Interdisziplinäre Kooperationen in den Natur- und Geisteswissenschaften wurden vergleichend betrachtet, in ihren je spezifischen Formen der Zusammenarbeit (Real 2012). ${ }^{8}$ Die direkte Zusammenarbeit zwischen Geistes- und Naturwissenschaften fand bislang vorwiegend in Beispielen aus dem 20. und 21. Jahrhundert Beachtung (Davidson \& Savonick 2017; Guerra 2008). Eine Ausnahme bildet die Technical Art History, die anhand von Beispielen die Zusammenarbeit von Kunsthistorikern, Künstlern und Chemikern Anfang des 19. Jahrhunderts und damit die Ursprünge der Konservierungswissenschaften untersucht (Dupré 2017; Bol 2017). Detailanalysen der Kooperationsform über die Grenzen der Wissenschaftskulturen hinweg liegen bisher jedoch nicht vor. Dies mag daran liegen, dass diese Form der Kooperation vergleichsweise selten und nur in Ausnahmefällen - wie im vorliegenden Beispiel - umfassend dokumentiert ist; zugleich sind diese Konstellationen besonders instruktiv. Unter anderem stellen sie besonders hohe Anforderungen an die erwähnte Integrationsexpertise. Wir werden daher besondere Aufmerksamkeit darauf richten, wie es gelang, die fachliche Expertise der Paläographen, Archäologen und Orientalisten mit der des Pflanzenphysiologen Wiesner zu verbinden. Es wird sich zeigen, dass auf beiden Seiten Integrationsexpertise vorlag, wobei Wiesner sich im Laufe der Zeit deutlich stärker auf die Paläographie zubewegte als anders herum.

Denn Wiesners Rolle in diesen Kooperationen änderte sich im Laufe der Zeit erheblich; damit eignet sich unser Beispiel auch dafür, Muster und Varianten der wissenschaftlichen Kooperation zu unterscheiden. So antwortete Wiesner anfangs nur reaktiv auf spezifisch formulierte Forschungsfragen und arbeitete seinem Kooperationspartner zu. Wir schlagen vor, in diesen Fällen von einer „geschlossenen Kooperation“ zu sprechen, die in klar vorgespurten intellektuellen Bahnen bleibt. Im weiteren Verlauf zeigte Wiesner zunehmend Eigeninitiative, wartete nicht mehr auf Aufträge, sondern begann, sich selbstverantwortlich und innovativ in die Zusammenarbeit einzubringen. Hier lässt sich von einer "offenen Kooperation“ zwischen Wiesner und den Paläographen sprechen: Beide Kooperationspartner entwickelten Methoden und Fragestellungen weiter, was neue, unvorhergesehene Forschungsrichtungen und Ergebnisse ermöglichte - auch über den Gegenstand der eigentlichen Kooperation hinaus. So bereicherte Wiesner die Paläographie um methodische Zugänge und papierhistorische 
Befunde, nutzte aber die mikroskopische Untersuchung der alten Manuskripte auch für seine botanischen Interessen.

Diese Entwicklung von einer geschlossenen Kooperation, in der Wiesner den Paläographen lediglich zuarbeitete, zu einer (ergebnis-)offenen Kooperation lässt sich auch damit erklären, so unsere These, dass Wiesner seine Integrationsexpertise zunehmend weiterentwickelte, das heißt ein immer besseres Verständnis dessen gewann, wie er seine eigenen botanischen und warenkundlichen Kenntnisse mit den Befunden und Interessen der historischen Papierforscher verknüpfen konnte. Doch wie wir sehen werden, blieb Wiesner auch dabei nicht stehen. Über die Integrationsexpertise hinaus erarbeitete er sich umfassende inhaltlich-sachliche Expertise der Papiergeschichte - bis an die Grenze der eigenen disziplinären Mobilität. Denn im Ergebnis seiner jahrzehntelangen Zusammenarbeit sah Wiesner sich schließlich selbst als Papierhistoriker, nicht mehr nur als interdisziplinär beitragender Experte. ${ }^{9}$ Betrachten wir nun die drei Phasen dieser Entwicklung am Fall.

\section{Pflanzenfasern und Paläographie - Wiesner und Karabacek}

In der Papyrus-Sammlung der kais. Hoheit Herrn Erzherzog Rainer befinden sich, wie ich Ihnen einmal schon mitzutheilen die Ehre hatte, auch Proben des ersten von den Arabern erzeugten Papieres (8-10 Jhdt.), von welchem ich vermuthe, daß sie aus Baumwollfasern hergestellt sind. Eine genauere Untersuchung derselben, welche Sie mir zuzusagen bereits die Güte hatten, würde über diese sehr wichtige Frage Licht verbreiten (Karabacek 1884: MA8, 3.5.83.A1.18, Hervorhebung im Original).

Dieser Brief des Wiener Orientalisten Josef Karabacek (1845-1918) aus dem Jahre 1884 an Julius Wiesner (1838-1916) dokumentiert die zwischen den beiden Wissenschaftlern getroffene Vereinbarung zur mikroskopischen Untersuchung von Papierproben. Es folgte eine mehrjährige Zusammenarbeit bis zum Jahre 1888 sowie zahlreiche weitere Briefe bis $1915 .^{10}$ Die Kooperation mit Karabacek markiert den Beginn von Wiesners Karriere in der Papiergeschichte. Nichts deutet darauf hin - etwa Bemerkungen in Briefen oder erhaltene Rechnungen -, dass Wiesner für diese Arbeit bezahlt wurde, seinen Beitrag also als Dienstleistung und Auftragsarbeit verstand. Von Anfang an war es ein Angebot zur wissenschaftlichen Kooperation, das Wiesner auch hätte ablehnen können. 
Wiesner blickte zu diesem Zeitpunkt bereits auf eine lange, erfolgreiche Karriere zurück (Wininger 1979: 282; siehe auch: Kisser 1963; Linsbauer 1917; Wurzbach 1888). Von 1872 bis 1909 hielt er an der Universität Wien den weltweit ersten Lehrstuhl für Pflanzenphysiologie, zugleich war er bis 1880 Professor für technische Warenkunde und technische Mikroskopie am Wiener Polytechnikum. Wiesner trug erheblich zur Entwicklung und Etablierung all dieser Fachgebiete bei. Er publizierte auf nahezu allen Bereichen der Pflanzenphysiologie und war aktiv daran beteiligt, den Gegenstandsbereich des noch jungen Faches zu umreißen (vgl. Wiesner 1877, 1881, 1892, 1896, 1904a, 1907). ${ }^{11}$ Sein pflanzenphysiologisches Institut galt als eines der „best eingerichteten seiner Art“ (Molisch 1916: 83). In diesem Institut führte Wiesner auch die mikroskopischen Analysen für Karabacek und seine späteren Kooperationspartner durch (Wiesner 1910: LHAS, 10/ fols. 115). Dabei hatte Wiesner sich schon vorher, in seiner Tätigkeit am Polytechnikum, als Experte für die mikrochemische Analyse von Pflanzenfasern und deren Verarbeitung zu industriellen Produkten wie Papier profiliert. Auch nach seiner Emeritierung mikroskopierte Wiesner noch in den Räumen des Instituts Papierproben, wie wir aus einem Brief von Wiesner an Aurel Stein erfahren:

Ich habe - dank dem freundlichen Entgegenkommen meines Nachfolgers im Amte, Prof. Molisch, der einer meiner fähigsten und liebsten Schüler ist - im pflanzenphysiol. Institute meinen eigenen Arbeitsraum und stehen mir die Mittel des Institutes weiter zur Verfügung, so dass ich meine Arbeiten, auch die mikroskopische, nach wie vor auszuführen in der Lage bin. (Wiesner 1910: LHAS, 10/fols. 120)

Wie und wo Wiesner und Karabacek sich kennenlernten, ist nicht genau zu bestimmen. Die Wiener Wissenschaftslandschaft um 1900 war klein und überschaubar, und die Professorenschaft in vielfacher Weise vernetzt - nicht zuletzt wurde die Philosophische Fakultät der Universität Wien erst 1975 in einzelne Fachbereiche geteilt (Mühlberger 2009: 97). ${ }^{12}$ Weiterhin waren Karabacek und Wiesner beide Mitglieder der Akademie der Wissenschaften in Wien und trafen demzufolge auch in diesem Kontext regelmäßig aufeinander. Es ist daher gut möglich, dass Karabacek um Wiesners Kompetenz als technischer Experte von Papier und Mikroskopie wusste und ihn persönlich auf diese Fragen ansprach.

Der eingangs zitierte Brief nimmt Bezug auf die „Sammlung Papyrus Erzherzog Rainer“ (PER). Diese umfasste etwa 10.000 Papyri und andere Papiere aus Ägypten (El-Fajiûm), die 1883 auf Karabaceks Initiative hin erworben und ins k.k. Österreichische Museum für Kunst und Industrie in Wien überführt worden waren (Karabacek 1883, 1885; Selander 2008). Sie ist heute Bestandteil der Papyrussammlung der Österreichischen National- 
bibliothek, eine der bedeutendsten und größten Manuskriptsammlungen der Welt. Karabacek war mit der Sichtung, Bearbeitung und wissenschaftlichen Auswertung der orientalischen Handschriften betraut. Er war Experte für arabische Münzen, Papyri und Textilien, Gründungsmitglied des orientalischen Instituts in Wien, Professor für die Geschichte des Orients und ihrer Hilfswissenschaften sowie später Direktor der Wiener Hofbibliothek. Karabacek war aber kein rein philologisch arbeitender Orientalist, sondern verband quellenkundliches mit materialkulturellem Interesse (Troelenberg 2011: 233; Hauser 2005: 77; Marchand 1994: 109; auch zu Karabacek: Ali-de-Unzaga 2012: 75-86; Becker 1920: 233-238; Bihl 2009; Gottschalk 1977; Mauthe 1999: 11-42; Rhodokanakis 1919: 188-198). In seiner Forschung interpretierte Karabacek Schrift- und Materialquellen unter Rückgriff auf ihren kulturhistorischen Kontext. So setzte er etwa in der Kooperation mit Wiesner die Materialität von Manuskripten mit der Geschichte des Papiers und seiner Herstellung in Beziehung.

Dieser „Einbezug von Realstudien in die orientalistische Leitdisziplin“ (Troelenberg 2011: 234) ist symptomatisch für eine breitere Transformation und Entwicklung der historischen Wissenschaften in der zweiten Hälfte des 19. Jahrhunderts. Teile der Altertumswissenschaften ergänzten zunehmend ihre philologische Ausrichtung mit einer Untersuchung materieller Überreste, und als Gegengewicht zur politischen Geschichtsschreibung richtete sich das Interesse auf eine weit gefasste Kulturgeschichtsschreibung (Hauser 2005; Mehr 2009; Schleier 2003). Eine Reihe historischer Disziplinen wie die Archäologie, die Kunstgeschichte und die Orientalistik erkannten, dass sie zur Beantwortung bestimmter Fragen mit Naturwissenschaftlern kooperieren mussten - insbesondere, wenn es um die materielle Dimension ihrer Forschungsgegenstände ging (Caley 1967: 121). ${ }^{13}$

Viele Beispiele dokumentieren den Aufstieg einer naturwissenschaftlichen Analyse archäologischer Artefakte im deutschsprachigen Raum um 1900. 1888 wurde in den königlichen Museen zu Berlin sogar ein eigenes chemisches Labor zur Untersuchung materieller Quellen eingerichtet, unter Leitung des Chemikers Friedrich Rathgen (1862-1942). Seine Hauptaufgabe war die Konservierung und Restaurierung archäologischer Artefakte, er gilt als der Vorreiter der Konservierungswissenschaft (Caldararo 1987; Clavir 1998; Corfield 1988; Plenderleith 1998; zu Rathgen auch: Bracchi 2014; Brittner 1943: 19-20; Gilberg 1987: 105-20; Hiecke 1942: 91; Riederer 1976). Rathgens Materialuntersuchungen dienten aber auch analytischen Fragestellungen (Unger \& Debbert 1988). Er untersuchte Tonscherben aus Babylon (Rathgen 1903, 1908), Glasperlen aus Ägypten (Rathgen 1913) und prüfte Gemälde und Skulpturen der Berliner Sammlung auf ihre Echtheit (Unger \& Debbert 1988). Etwa zur gleichen Zeit bemühte man sich in Berlin auch um die Analyse von Glasuren aus Assur. Walter Andrae 
(1875-1956), Kurator der Vorderasiatischen Abteilung der Berliner Museen, beauftragte damit das Preußische Materialprüfungsamt (Andrae 1923; Bär 2003). Der Wiener Kunsthistoriker Josef Strzygowski (1862-1941) versuchte den Einfluss Persiens auf chinesische Textilkunst nachzuweisen und bat dafür den uns bereits bekannten Pflanzenexperten Wiesner um die Analyse von Seidenstoffen (Stein 1911: LHAS 9/fols. 55; Strzygowski 1911: LHAS 9/fols. 69 und LHAS 9/fols. 78; siehe auch: Marchand 1994).

Die Anwendung naturwissenschaftlicher Analysen veränderte maßgeblich die Ausrichtung einiger historischer Disziplinen. Dies gilt auch für die Paläographie, die sich neuen Forschungsansätzen und Methoden öffnete (Härtel 2002; Henning 2000; Koch 2005). Die Zusammenarbeit von Wiesner und Karabacek bietet insofern auch Einblicke in die Geschichte der historischen Hilfswissenschaften, die im späten 19. Jahrhundert ihre Rolle im akademischen Betrieb zu definieren suchten. Die gezielte Anfrage von Karabacek deutet darauf hin, dass er wusste, was er von Wiesner zu erwarten hatte und wie er diesen Input mit seinen Befunden zu kombinieren gedachte. Neben einer weitgehenden Offenheit gegenüber naturwissenschaftlichen Daten ist dies Zeichen erheblicher Integrationsexpertise: Karabacek trat gut vorbereitet in diese interdisziplinäre Zusammenarbeit ein.

Fragen zur materiellen Grundlage von Papier stellten sich in Wien mit besonderer Dringlichkeit: Wien wurde ab den 1870er Jahren „geradezu überschwemmt mit den Produkten des Orients“ (Lessing 1900: 22), und dazu zählten auch die zahlreichen Papyri und andere Manuskripte aus ElFajiûm, die Wiesner untersuchte. Die Sammlung PER entwickelte sich zu einer der umfangreichsten Papyrussammlungen der Welt. Die Bedingungen für die materielle Analyse dieser Papiere waren nahezu optimal: 1854 war in Wien das Institut für österreichische Geschichtsforschung (IÖG) gegründet worden, im Bestreben, die historischen Hilfswissenschaften auf eine neue Grundlage zu stellen und zu professionalisieren (Lhotsky 1954). Dies erwies sich als überaus erfolgreich, und das IÖG war bald für seine Untersuchungen europaweit bekannt und vernetzt. Mit dem Direktor, Theodor Sickel (1826-1908), pflegte Wiesner sehr gute Beziehungen, auch begünstigt durch die räumliche Nähe ihrer Institutionen (zu Sickel: Erben 1926; Redlich 1927; zum Verhältnis von Wiesner und Sickel: Musil-Gutsch 2020). Darüber hinaus war Wiesner mit führenden technischen Papierexperten im deutschsprachigen Raum vernetzt, so korrespondierte er etwa mit Wilhelm Herzberg (1861-1936) (Herzberg 1893: MA8, 3.5.83.A1.18). Seit 1884 leitete Herzberg die Abteilung für Papierprüfung der Königlichen Mechanisch-Technischen Versuchsanstalt in Berlin (ab 1900: Königliches Materialprüfungsamt; vgl. Ruske 1971, 79.). 
Zurück zur Anfrage von Karabacek. Ihn interessierten vor allem Proben der sehr frühen, von Arabern hergestellten Papiere der Sammlung PER aus dem 7. und 8. Jahrhundert n. Chr. Karabacek vermutete, dass diese Proben unverarbeitete Baumwollfasern enthielten. Damit bargen sie für ihn das Potenzial, eine in der Paläographie der Zeit „lebhaft ventilirte“ (Wiesner 1887: 180) Frage zu beantworten: $\mathrm{Ob}$ das älteste, zu jener Zeit bekannte Papier wirklich aus roher Baumwolle gefertigt worden war oder aber aus Hadern bestand, also Textilresten, dem vor allem im europäischen Spätmittelalter gebräuchlichen Schreibstoff (Lupi 1875; Briquet 1884). Die Datierung des ältesten bekannten Papiers war auf diese Weise eng verbunden mit der Frage nach den kulturhistorischen Anfängen der Papierherstellung; davon abgeleitet konnten auch jüngere Papiere datiert werden, was wiederum eine genauere zeitliche Einordnung von Textquellen erlaubte.

Die These vom Papier aus roher Baumwolle stammte aus den Anfängen der Paläographie im 18. Jahrhundert, vorgebracht von namhaften Pionieren des Faches wie zum Beispiel Lodovico Antonio Muratori (1672-1750) und Bernard de Montfaucon (1655-1741). Seither hatte sich diese These im Feld gehalten und war zumeist ungeprüft übernommen worden. Doch seit den 1870er Jahren wurde in der Paläographie vermehrt daran gezweifelt, denn überzeugende Belege für die Fertigung von Papier aus unverarbeiteter Baumwolle gab es nicht - insbesondere gab es keine Bestätigung für die Existenz dieses Papiers auf Grundlage einer botanisch sachkundigen, mikroskopischen Untersuchung der Fasern. Bislang hatte man entweder von Bezeichnungen in historischen Quellen auf das Material einer Handschrift geschlossen oder eine Materialbestimmung mit bloßem Auge vorgenommen, das heißt die Optik der Gewebestruktur in durchscheinendem Licht geprüft (Wiesner 1887: 189; Sickel 1872: 446). Um die tatsächliche Beschaffenheit der ältesten Papiere zu klären, war diese Vorgehensweise unzureichend.

Karabacek und andere erkannten, dass „[h]ier [...] also die Grenzscheide [lag], wo der Historiker vor dem Physiologen sich zurückziehen musste“ (Karabacek 1885: 165). Die Anfrage von Karabacek an Wiesner war dabei nicht die erste ihrer Art. An verschiedenen Standorten in Europa bildeten sich gegen Ende des 19. Jahrhunderts Zweiergespanne aus Paläographen und Naturwissenschaftlern, die in altem Papier nach Baumwollfasern suchten. Der Schweizer Papierhändler und -forscher Charles-Moïse Briquet (1839-1918) etwa versuchte gemeinsam mit dem Pharmakologen Jaques Brun (1826-1908) die Baumwollfrage zu beantworten. Er stand darüber mit Wiesner in Kontakt und berichtete ihm von weiteren Kooperationen:

Il est très curieux qu'une recherche de ce genre ait été poursuivre presque simultanément et sans que de part ou d'autre, nous en eussions réciproquement connaissance, par vous, a Vienne, par MM les profs 
Girard et Giry à Paris, et par moi à Genève. Que nous soyons tous arrivés au même résultat, cela fait honneur au microscope. (Briquet 1886: MA8, 3.5.83.A1.18) $)^{14}$

Briquet berichtete Wiesner von der Zusammenarbeit des französischen Paläographen Arthur Giry (1848-1899) mit dem Chemiker Aimé Girard (1830-1898) (siehe auch o. A. 2019; Demeulenaere-Douyère \& François 2019). Unerwähnt ließ er den italienischen Paläographen Clemente Lupi (1840-1919), auf den die ersten Versuche zur mikroskopischen Analyse von Papier mit Botanikern zurückgehen (Capannelli \& Insabato 2000: 166-168; Capannelli \& Lenzi o. J.; Pagliai 1919). Lupi arbeitete bereits 1872 zu diesem Zweck mit Teodoro Caruel (1830-1898) und Antonio Mori (1847-1902) zusammen (Wiesner 1902: 3; Lupi 1875: 45-46). Diese interdisziplinären Zusammenschlüsse sind Beispiele für geschlossene Kooperationen, wie sie eingangs eingeführt wurden: Die Paläographen stellten eine Frage, die von den Naturwissenschaftlern mit Hilfe mikroskopischer Methoden zu beantworten war, nämlich: Gibt es in diesem Papier rohe Baumwollfasern oder nicht? Die Antwort lautete: ja; aber wie Wiesner zeigen konnte, hatten seine Vorgänger unzureichende Methoden verwendet und die Ergebnisse falsch ausgewertet, da es ihnen an Hintergrundwissen mangelte. ${ }^{15}$ Wir haben zudem keine Hinweise darauf, dass sich in diesen Konstellationen die Naturwissenschaftler für die epistemischen Ziele der Paläographen interessierten oder gar eigene Fragestellungen an das Material verfolgten. Es waren klare Fälle geschlossener Kooperationen, deren Ergebnisse, wie sich zeigen sollte, die Frage nach dem Baumwollpapier nicht umfassend beantworteten.

In ihrer Anfangsphase folgte die Kooperation von Wiesner und Karabacek demselben Muster. Karabacek trat mit einer konkreten Frage zur Papierzusammensetzung der arabischen Manuskripte an Wiesner heran: „Baumwolle oder Hadern?"; und zwischen diesen Alternativen sollte Wiesner entscheiden. Wiesner griff dafür auf seine Kenntnis botanischer und warenkundlicher Verfahren zurück und nutzte sie zur Analyse des alten Papiers. In der Veröffentlichung eines Zwischenstands seiner Untersuchungen von 1886 erklärte Wiesner: „Alle mir bisher übergebenen Objecte bestehen im Wesentlichen aus demselben Fasernmateriale [...] und wurden, allem Anscheine nach, auf gleiche Weise erzeugt. [...] Diese Papiere sind durchwegs aus Hadern (Lumpen) bereitet" (Wiesner 1887: 45). Damit widersprach sein Befund der ursprünglichen Annahme von Karabacek - und den naturwissenschaftlichen Experten vor Wiesner.

Die umfassenden Abhandlungen erschienen 1887: Karabacek veröffentlichte einen Aufsatz über „Das arabische Papier“ (1887) und Wiesner seine „Faijûmer Papiere“ (1887). Die Publikationen waren zwar - wie beide Wis- 
senschaftler oft betonten - unabhängig voneinander entstanden, aber eng aufeinander bezogen. Die Abhandlungen erschienen zudem in derselben Zeitschrift, nämlich den „Mitteilungen der Sammlung Papyrus Erzherzog Rainer" (Karabacek 1887; Wiesner 1887). Dabei folgte der Aufsatz von Wiesner direkt demjenigen von Karabacek, quasi als technischer Appendix. ${ }^{16}$ Die Tatsache, dass es nicht zur Ko-Autorenschaft kam, sollte nicht überbewertet werden (Csiszar 2018). ${ }^{17}$ Während wissenschaftliche Kooperation sich im 20. und 21. Jahrhundert vor allem in gemeinsamen Publikationen manifestiert (Wray 2006), gilt dies für das 19. Jahrhundert nicht in gleicher Weise. Die hier gewählte Form der Publikation dokumentiert aber, dass es sich um eine geschlossene Kooperation mit klar definierter Hierarchie handelte - Karabacek ging voran und formulierte die historische Interpretation, Wiesner ergänzte die Datengrundlage.

Erst in späteren Phasen der Zusammenarbeit publizierte Wiesner eigenständig zu seinen papierhistorischen Materialuntersuchungen (Wiesner 1902, 1911). Schon die „Faijûmer Papiere“ (1887) zeigen aber, dass Wiesner nicht bei der Bearbeitung der ihm zugedachten Frage stehen blieb, sondern den Gegenstand aus seiner eigenen Perspektive und Sachkenntnis heraus neu durchdachte. Wiesner untersuchte nicht nur die arabischen Papiere aus der Sammlung PER, sondern zahlreiche weitere orientalische und europäische Papierproben, die ihm von namhaften Paläographen aus ganz Europa zugeschickt wurden (Wiesner 1887: 255, 260). ${ }^{18}$ Unterstützung erfuhr er dabei vor allem durch Theodor Sickel, den bereits erwähnten Direktor des IÖG, der über sein hervorragendes Netzwerk Papierproben aus acht italienischen Archiven ${ }^{19}$ für Wiesner beschaffen konnte (Wiesner 1887: 260; ÖNB, 567/2-4). ${ }^{20}$ Keine einzige dieser Proben bestand aus roher Baumwolle. Dieser Befund diente Wiesner als Grundlage, um die These vom Baumwollpapier zurückzuweisen und eigenverantwortlich Aussagen über historisch relevante Fragen zu treffen.

Wiesner untersuchte zudem nicht nur die Art der Pflanzenfasern, wie ursprünglich von Karabacek beabsichtigt. Unter Rückgriff auf seine spezifische, auch warenkundliche Expertise prüfte er zahlreiche weitere Bestandteile des Papiers, die in der Paläographie bis dahin nur wenig Aufmerksamkeit erfahren hatten: die zwischen das Fasergewebe gedrückte Füllung des Papiers, die Inkrustierung, die Länge der Fasern, die Tinte, den Staub, der den Papieren anhaftete, sowie die Leimung der Papiere. Gerade dieser letzte Punkt erwies sich als aufschlussreich. Indem Wiesner die Papiere systematisch einer chemischen Analyse unterzog, fand er heraus, dass alle Papiere bis zum Ende des 13. Jahrhunderts mit Stärkekleister geleimt waren. ${ }^{21}$ Erst danach wurde Tierleim verwendet. ${ }^{22}$ Wiesner wandte zur Beweisführung zwei einfache chemische Testverfahren an: Die Jod-Stärke Reaktion und die „Millon-Reaktion“. Verfärbte sich eine mit einer Jodlösung 
beträufelte Papierprobe bläulich, so war dies der Nachweis dafür, dass das Papier Stärke enthielt. Verfärbte sich das Papier rot beim Aufträufeln einer Lösung von Quecksilbernitrat in salpetriger Säure, zeigte dies Proteine an und somit tierischen Leim.

Gerade das Beispiel der eigeninitiativ angewandten chemischen Methoden zur Untersuchung der Leimung zeigt, dass Wiesner seine Integrationsexpertise im Laufe der Zeit erheblich erweiterte - insbesondere mit Blick auf die hohe Bedeutung einer unabhängigen Methode der Datierung für die Paläographie. Für seinen Beitrag in der Zusammenarbeit hatte Wiesner die Datierung des Papiers durch die Paläographen übernommen, mit den Resultaten seiner Materialanalysen verknüpft und eine neue Korrelation von Datierung und Leimung etabliert. So konnte Wiesner eine weitere bisher unhinterfragt übernommene Grundannahme der Papiergeschichte widerlegen; denn bis zu diesem Zeitpunkt hatte Tierleim als das älteste Leimungsmaterial gegolten. Diese Einsicht hatte direkte Auswirkung auf die Paläographie. Ausgehend von Wiesners Befund, dass sich die Leimung europäischer Papiere vom 13. ins 14. Jahrhundert wandelte, ließ sich nun die Datierung mittelalterlicher Handschriften und Urkunden unabhängig von ihrem Inhalt überprüfen (Mühlbacher 1888: 481).

Tatsächlich führte das von Wiesner entdeckte Leimungskriterium da$\mathrm{zu}$, dass Handschriften umdatiert wurden. So hatte Wiesner in einer hebräischen Handschrift aus der Pariser Nationalbibliothek tierischen Leim nachgewiesen (Wiesner 1887: 256); in der Folge wurde die bisherige Datierung (auf das Jahr 1271) korrigiert und ein paar Jahrzehnte später angesetzt (Mühlbacher 1888: 481). Wiesners Analyse der Papierleimung zeigt insofern den Mehrwert einer offenen Kooperation. Beide Seiten konnten ihre Kenntnisse, Fähigkeiten und Interessen einbringen, und in der Folge ging das Ergebnis weit über die ursprüngliche Frage an Wiesner hinaus. Karabacek war gar nicht auf die Idee gekommen, Wiesner nach Leimungen, Füllung und anderen Details zu befragen; Wiesners Befunde dazu waren jedoch für ihn von höchstem Interesse. Zudem sieht man die Bedeutung der Integrationexpertise; denn nur indem Wiesner bereit war, sich in Forschungsfragen der Paläographen hineinzudenken, konnte er seine eigenen Ansätze dazu produktiv in Beziehung setzen.

Wiesners Forschung, vor allem die Widerlegung der These vom Baumwollpapier, wurde mit Begeisterung aufgenommen. ${ }^{23}$ So rückte sogar der deutsche Paläograph Wilhelm Wattenbach (1819-1897), ein Vertreter der Baumwollpapierthese (Wattenbach 1875: 92-93), von seinem ursprünglichen Standpunkt $\mathrm{ab}^{24}$ und bezeichnete Wiesners „Faijûmer Papiere“ (1887) als „epochemachend“ (Wattenbach 1888: 667; zu Wattenbach: Löwenfeld 1889; Rodenberg 1898; Zeumer 1889). Bemerkenswert ist Wiesners Beitrag jedoch nicht nur durch seine sachkundige Analyse sämtlicher Pa- 
pierbestandteile. Wiesner hatte sich überdies kritisch mit dem Stand der Forschung in Paläographie und Papiergeschichte auseinandergesetzt und verstand es, sich darin zu positionieren. So hatte er festgestellt, dass Paläographen durchaus botanische Werke konsultierten (Wattenbach 1875). Zur Baumwoll- und Bastfaserunterscheidung in alten Handschriften verwendeten sie etwa die Bücher von Matthias J. Schleiden (1804-1881) oder Siegfried Reissek (1819-1871) und griffen auf die darin angegebenen Kennzeichen für Pflanzenfasern zurück. Aufgrund seines eigenen, inzwischen umfassenden Papierwissens befand Wiesner diese Methode als zutiefst unzureichend für paläographische Materialuntersuchungen: Botaniker wie Schleiden zogen nur unverletzte Pflanzenfasern als Referenz heran - bei der Papierherstellung wurden Pflanzenfasern aber gequetscht und zerstampft, und sie veränderten sich dadurch grundlegend in ihrem Erscheinungsbild. Dies war bei der mikroskopischen Analyse zu berücksichtigen. ${ }^{25}$ So konnte man in aufgelockertem Zustand die Baumwollzellwand leicht mit der Wand einer zerstampften Lein- oder Hanfbastzelle verwechseln. Wiesner nutzte daher ergänzend mikrochemische Methoden. So wurde etwa durch Zugabe von Kupferoxyd-Ammoniak ${ }^{26}$ die Zellulose aufgelöst; die charakteristische Kuticula, also die äußere Schutzhaut der Zellwand, blieb jedoch unversehrt. Diese konnte Wiesner dann unter dem Mikroskop prüfen und eindeutig zuordnen (Wiesner 1877: 215).

Der solchermaßen kritische Kommentar Wiesners zu den bisherigen Methoden der Paläographie unterstreicht die Dynamik offener Kooperationen. Sie implizieren ein Gespräch auf Augenhöhe, in dem die Kooperationspartner gleichermaßen in ihrer Expertise ernst genommen wurden. In der botanischen wie auch technischen Mikroskopie und Faserkunde hatte Wiesner seinen Kollegen aus der Paläographie etliches voraus. Für die Sachfrage war es insofern gewinnbringend, seine Einschätzung zu hören und die bisherige Praxis zu revidieren. Dass sich die Paläographen in diesem Zusammenhang von Wiesner kritisieren lassen mussten, war Teil des Forschungsprozesses und wurde akzeptiert.

\section{Hadernpapier in Ostturkestan - Wiesner und Hoernle}

Wiesners Untersuchungen mit Karabacek wurden europaweit in paläographischen Fachkreisen bekannt; und am 19. Februar 1900 trat der britischdeutsche Orientalist Rudolf Hoernle an Wiesner heran mit dem Gesuch, „die materielle Untersuchung der ostturkestanischen Papiere zu übernehmen“ (Wiesner 1902: 3). ${ }^{27}$ Dabei ging es zunächst um die berühmten altindischen Bower-Manuskripte, die Hoernle als Indologe untersuchte (Wil- 
helm 1972: 362; zu Hoernle auch: Grierson 1919: 114-24; Sims-William 2012). ${ }^{28}$ Weiterhin sollte Wiesner Fragmente von Papierfunden der ersten zentralasiatischen Expedition 1900-1901 unter Leitung von Aurel Stein untersuchen, der dritte Kooperationspartner Wiesners, auf den wir im nächsten Abschnitt zurückkommen. Wiesner nahm den Auftrag an und veröffentlichte seine Ergebnisse zu Hoernles Proben in der Abhandlung "Ostturkestanische Papiere“ (1902). Es war das erste Mal, dass die Materialität alten chinesischen Papiers untersucht wurde; zudem handelte es sich um die ältesten bis dahin bekannten Papiere. Sie waren sogar älter als die arabischen Papiere des Fundes von El-Faijûm (Needham \& TsuenHsuin 1985: 11).

In der Kooperation mit Hoernle stand Wiesner vor einer komplexeren Ausgangslage als bei den Faijûmer Papieren, denn die zu prüfenden $\mathrm{Pa}-$ piere waren sehr unterschiedlicher Provenienz - nämlich indischen, chinesischen, persischen und islamitischen Ursprungs. Bei dem arabischen Papier hatte Wiesner eine klare Fragestellung zu lösen („Hadern- oder Baumwollpapier?"). In diesem Fall fehlten sowohl Auftrag als auch Arbeitshypothese. Schon mit seiner Anfrage trat also Hoernle mit Wiesner in eine offene Kooperation ein, indem er ihm ohne konkret vorgegebenes Erkenntnisziel Material zur Untersuchung überließ. Hoernle wusste, was er von der Kooperation erwarten konnte: eine umfassende Untersuchung mit den Methoden der Mikroskopie, Pflanzenphysiologie und Warenkunde sowie die Kompetenz, dieses Können und Wissen integrativ in die Kooperation einzubringen.

Wiesner musste für seine Analyse die mögliche Herkunft der Pflanzenfasern erst ermitteln; von Hoernle erhielt er allenfalls vage Anhaltspunkte zu den Rohmaterialien chinesischer Papiere (Wiesner 1902: 8). ${ }^{29}$ So begann Wiesner eine umfassende Erhebung aller in Indien, China und im arabischen Raum nachweislich zur Papierherstellung genutzten Pflanzen sowie aller weiteren Faser- und Kulturpflanzen Turkestans. Nicht für alle diese Fasern lagen verlässliche Angaben zu ihren mikroskopischen Kennzeichen vor; in solchen Fällen musste Wiesner diese Eigenschaften selbst durch histologische Faserstudien erheben (Wiesner 1902: 4). Davon zeugt etwa ein Brief, den Wiesner am 3. Februar 1902 an Hoernle schrieb:

Sie werden aus dem übersendeten Bogen auch ersehen haben, dass nun bezugs Untersuchung der ostturkestanischen Papiere gut vorgearbeitet ist. Denn jetzt können wir die Broussonelia-Edgeworthia-Reis und Bambusfaser mit Sicherheit in den Papieren nachweisen und von ihnen vermeintliche Hadern oder Lumpen Papiere genau unterscheiden. Diese Vorarbeit war aber dringend nötig. (Wiesner 1902: Bodleian, Ms Stein 458, fols. 76-77) 
Schließlich konnte Wiesner eine Liste von Fasern erstellen, die für die Analyse des Papiers zu berücksichtigen waren (Wiesner 1902: 9). Von diesen Studien profitierte auch seine pflanzenphysiologische und warenkundliche Forschung. So veröffentlichte Wiesner die mikroskopischen Charakteristika der oben genannten Pflanzenfasern, die Fasern der Edgeworthia sowie Bambusrohrfasern, in der zweiten Auflage seines Werkes „Rohstoffe des Pflanzenreichs, Bd. 2“ (Wiesner 1902: 9). Weiterhin nutzte er die „Ostturkestanischen Papiere“ (1902) als Plattform zur Publikation der von ihm bestimmten Kennzeichen von vier Faser-Typen: der Schilfrohr- und der Stipa-Faser sowie der Faser von Calamus Rotang und Daphne cannabina (Wiesner 1902: 10). Es war nicht das erste Mal, dass Wiesner auch als Naturwissenschaftler von seiner Kooperation mit Paläographen profitierte. Bereits während seiner Arbeit für die „Faijûmer Papiere“ (1887) hatte er aufgrund seiner Studien des alten Papiers die Methoden und Techniken der technisch-industriellen Papierprüfung weiterentwickelt und verbessert. ${ }^{30}$

Nach diesen Vorarbeiten, das heißt der Ermittlung der in Frage kommenden Pflanzenfasern und ihrer mikroskopischen Kennzeichen, verlief die Untersuchung in den "Ostturkestanischen Papieren“ (1902) methodisch nach demselben Prinzip wie jene der „Faijûmer Papiere“ (1887). Wieder präsentierte Wiesner detaillierte Angaben zu den Pflanzenfasern, zum Herstellungsverfahren und zur Leimung der Papiere. Die Befunde wurden dabei zunächst disziplinär nach Botanik und Paläographie getrennt: Der Beschreibung allgemeiner, distinkter Merkmale einzelner Fasern und ihrer entsprechenden Behandlung folgte eine Ergebnisübersicht der Papieranalysen. Im Verlauf der Kooperation hingegen verknüpfte Wiesner seine Materialanalysen mit eigenen historischen Fragestellungen - in der Rolle eines Papierhistorikers, der auf materieller Quellenbasis argumentierte. Von Integrationsexpertise ist in diesen Fällen nicht mehr zu sprechen; vielmehr wurde die interdisziplinäre, hoch integrierte Kooperation für Wiesner zum Katalysator disziplinärer Mobilität.

Überraschenderweise fand Wiesner Hadernbestandteile in den ostturkestanischen Papieren. Bislang führte man die Erfindung des Hadernpapiers auf den arabischen Raum zurück, während man die Anfänge der Papierherstellung aus Seide oder Hanf in China verortete und auf den Anfang des 2. Jahrhunderts n. Chr. datierte. In einem Brief an Hoernle schrieb Wiesner, dass er "mit aller Bestimmtheit“ sagen könne, dass es sich bei einem von ihm untersuchten chinesischen Papier aus dem 6. oder 7. Jahrhundert um ein mit Stärkekleister geleimtes Hadernpapier handele, ganz ähnlich den alten arabischen Papieren (Wiesner 1902: Bodleian, Ms Stein 458, fols. 77). Briefe und Publikationen von Wiesner aus dieser Zeit zeugen davon, dass er sich vermehrt den historischen Implikationen seiner Untersuchungen zuwandte. Der Fund von Hadern in chinesischen Papie- 
ren deutete für Wiesner und Hoernle darauf hin, dass bereits im China des 2. Jahrhunderts n. Chr. Hadernbestandteile zu Papier verarbeitet wurden. ${ }^{31}$ Dieses Verfahren war dann vermutlich von den Arabern weiterentwickelt worden, bis es schließlich zur Herstellung von reinem Hadernpapier kam, wie es im europäischen Mittelalter gebräuchlich war. Würde man allerdings reines Hadernpapier chinesischen Ursprungs finden, aus einer Zeit vor dem arabischen Hadernpapier, wäre damit der Nachweis erbracht, dass die Chinesen auch dafür als die eigentlichen Erfinder gelten müssten.

Die historische Dimension seiner Ergebnisse war Wiesner bewusst; zudem war ihm bewusst, dass er sich damit auf heikles Terrain begab. Denn Karabacek, Wiesners Kollege und ehemaliger Kooperationspartner, hielt die Araber für die unstrittigen Erfinder des Hadernpapiers. Karabacek hatte deshalb Einwände gegenüber Wiesners Befunde, wie Wiesner in einem Brief an Hoernle berichtete:

Denn wie Karabacek sagt, haben die Araber schon 750 Hadernpapier erzeugt. Er meint mit Rücksicht auf damaligen Verkehre wäre es doch möglich oder schon wahrscheinlich, dass das Papier von den Arabern erzeugt wurde und später chinesisch beschrieben wurde. Nach Kar. haben die Chinesen [...] im J. 940 n. Chr. aus Lumpen Papier erzeugt $\mathrm{zu}$ welcher Zeit das Hadernpapier schon in Europa und im Orient benutzt war und erzeugt wurde. Doch das sind Dinge, die sich meinem Urtheil entziehen. (Wiesner 1902: Bodleian, Ms Stein 458, fols. 77)

Wiesner begann also, eigene Hypothesen $\mathrm{zu}$ formulieren und papierhistorische Zusammenhänge mit Hoernle und Karabacek zu besprechen. Zwar war er zunächst noch bereit, wie aus obigem Zitat hervorgeht, in historischen Fragen den Experten der Disziplin den Vorrang zu lassen. Mit Blick auf seine naturwissenschaftlichen Befunde hingegen ließ er sich durch Karabacek nicht beirren:

Nach reiflicher Überlegung bin ich zu der Meinung gelangt, mich durch eine etwaige Kontroverse über paleographische Fragen nicht korrigieren $\mathrm{zu}$ lassen und die Resultate meiner mikroskopischen Untersuchung in den Dienst der historischen Forschung zu stellen und die Zugehörigkeit der Datierung ganz den Historikern anheim gebe. (Wiesner 1902: Bodleian, Ms Stein 458, fols. 79) 


\section{Hadernpapier in China - Wiesner und Stein}

Von dieser Haltung, in historischen Fragen der Datierung und Hypothesenbildung anderen den Vortritt zu lassen, ist nicht mehr viel zu spüren in der dritten Kooperationsbeziehung, die hier betrachtet werden soll: Wiesners Zusammenarbeit mit Aurel Stein (1862-1943) aus den Jahren 1902-1904, in denen Wiesner weiter an den asiatischen Papieren forschte, die Steins Grabungen zutage förderten. ${ }^{32}$ Stein übermittelte ihm Papierproben tibetanischer und chinesischer Manuskripte zur Analyse; und angesichts seiner Befunde in diesen Fällen kam Wiesner zu dem Ergebnis,

dass die datierten Chines. Mrkpte zu denselben Resultaten führten, wie die [...] von Prof. Hoernle zur Untersuchung überlassenen. Ich glaube, dass meine Aufstellungen durch die Untersuchung Ihres [Anm.: Steins] kostbaren Materials eine neue starke Stütze gewonnen haben. Ich für meine Person kann an der Richtigkeit meiner Aufstellung über den Entwicklungsgang der Papiererzeugung nicht zweifeln. (Wiesner 1903: Bodleian, Ms Stein 112, fols 49)

Für Wiesners Rolle als Kooperationspartner war dies ein entscheidender Wendepunkt. Die Untersuchungen für Stein belegten seine Annahmen zum Hadernpapier mit zusätzlichem Quellenmaterial - er war nunmehr überzeugt, dass er recht hatte (und nicht Hoernle oder Karabacek). Wiesner emanzipierte sich von seinen Kooperationspartnern, indem er zur eigenen Hypothesenbildung auf dem Feld der Papiergeschichte überging. Zwar arbeitete er schon lange nicht mehr den Paläographen in geschlossener Kooperation zu, wie einst bei Karabacek; aber auch in der offenen Kooperation mit Hoernle waren die Resultate noch geteilt präsentiert worden: einerseits Wiesners naturwissenschaftlich erhobenen Befunde zu Pflanzenfasern und Papier, andererseits die Interpretation durch die Historiker. Dies war vor allem deswegen möglich, weil Wiesner zunehmend kompetenter wurde, die Felder geeignet zu verknüpfen. Aber in seiner Kooperation mit Stein sprach Wiesner nicht mehr als Botaniker. Er sprach als Papierhistoriker eigenen Rechts, der seine Daten selbst auswertete und auf dieser Grundlage eigenverantwortlich eine Hypothese zur Entwicklung des Papiers verteidigte. Die Ergebnisse präsentierte Wiesner in einem Aufsatz, der aus der Zusammenarbeit mit Stein in den Jahren 1902-1904 hervorging: „Ein neuer Beitrag zur Geschichte des Papiers“ (Wiesner 1904b: 9-23). Hierin widersprach Wiesner nun offiziell der historischen Fachmeinung von Karabacek, als gleichberechtigter Diskussionspartner auf dem ihm ursprünglich fremden Feld der Paläographie.

Die Titel von Wiesners weiteren Publikationen markieren diese Wende. Betrachtet man die Hauptpublikationen bisher, so hatte er diese eingeleitet 
als „Resultate naturwissenschaftlicher Untersuchungen, welche allerdings das Ziel verfolgten, einen Beitrag zur Geschichte des Papiers zu liefern“ (Wiesner 1904b: 2). Sie trugen dementsprechend Untertitel, die auf die naturwissenschaftlich-technische Relevanz der Studien verwiesen. Das änderte sich nun: Weder „Ein neuer Beitrag zur Geschichte des Papiers“ (1904), noch die darauffolgende Publikation „Hadernpapiere“ (1911) gaben vor, einen Beitrag zur technischen Papierprüfung oder zur Mikroskopie mit technischen oder naturwissenschaftlichen Zusatzstudien zu leisten. Es ging Wiesner bei seinem „Beitrag zur Geschichte des Papiers“ (1904) ausdrücklich darum, eine „historische Untersuchung“ zu veröffentlichten, die „für den Naturforscher als solchen kaum ein Interesse [hat], wohl aber für den Historiker und [...] den Zwecken des Paläographen" dient; und die deshalb auch in den Schriften der philosophisch-historischen Klasse der Wiener Akademie der Wissenschaften publiziert wurde (Wiesner 1904b: 2-3.).

Auch die Praktiken der Kooperation hatten sich geändert. In der Zusammenarbeit mit Stein war Wiesner nicht mehr nur Empfänger von Material, das sein Kooperationspartner ihm übersandte. Vielmehr ergriff selbst Wiesner die Initiative und bat seinen Kooperationspartner Stein, ihm bestimmtes Material zu schicken. Dabei handelte es sich um Rohmaterial, das seine „Studien zur Geschichte der ostturkestanischen Papiere sehr fördern“ würde und mit Hilfe dessen Wiesner „erfahren könnte, aus welchem Materiale derzeit in Ostturkestan (besonders in Khôtan) Papier erzeugt wird“ (Wiesner 1903: Bodleian, Ms Stein 112, fols. 36-37). Wiesner gab Stein dabei genaue Anweisungen zum Zustand der Pflanze (beblättert und mit Früchten) und zum Transport (in einem Buch getrocknet, durch ein Gewicht beschwert) (Wiesner 1903: Bodleian, Ms Stein 112, fols. 36-37). $\mathrm{Zu}$ bedenken ist dabei immer, dass Wiesners mikroskopischen und chemischen Untersuchungen Proben des kostbaren Materials zerstörten. Dass er dennoch mit seinen Anfragen erfolgreich war, bezeugt eindrucksvoll, dass Wiesners neu gewonnenes Selbstbewusstsein zumindest von Stein als legitim anerkannt wurde.

Zwar konnte Wiesner in den „Ostturkestanischen Papieren“ (1902) und dem „Beitrag zur Geschichte des Papiers“ (1904) zeigen, dass Hadernpapier bereits im chinesischen Raum erzeugt worden war. Die Frage, ob die Chinesen die tatsächlichen Erfinder des Hadernpapiers gewesen waren, hatte sich allerdings nicht final klären lassen, da man unter den bislang untersuchten chinesischen Papieren kein reines Hadernpapier gefunden hatte. Auf der zweiten seiner vier großen Expeditionen nach Zentralasien in den Jahren 1906-1908 fand Stein weitere Manuskripte, die er ebenfalls von Wiesner untersuchen ließ. In dieser zweiten Episode seiner Zusammenarbeit mit Stein in den Jahren $1910-1911^{33}$ baute Wiesner seine Auto- 
rität als naturwissenschaftlich arbeitender Papierhistoriker weiter aus: Er unternahm auch in diesem Fall eigene Nachforschungen, formulierte abermals historische Hypothesen und prüfte diese eigenständig am Material. Diese Untersuchungen waren längst nicht mehr Mittel zum Zweck oder ein randständiges Nebenprojekt; wie aus dem ersten Antwortschreiben an Stein hervorgeht, war Wiesner inzwischen von persönlichem Interesse an historischen Fragestellungen motiviert:

Selbstverständlich wird es, wie früher, mir große Freude bereiten, Ihnen dienen zu können; ich erkläre mich mit Vergnügen bereit, die gewünschten mikroskopischen Papieruntersuchungen auszuführen: Sie wissen ja, wie lebhaft mich die Frage der Geschichte des Papieres interessiert. (Wiesner 1910: LHAS, 10/fols. 115)

Die von Stein ausgegrabenen Manuskripte waren sehr alt - sie wurden auf das 2. Jahrhundert n. Chr. datiert. Diese Angaben bekam Wiesner von Stein übermittelt, und das erinnert daran, dass der Informationsaustausch weiterhin in beide Richtungen lief. Wiesner erforschte zwar nun eigenständig historische Fragestellungen auf der Grundlage des von ihm analysierten Materials. Aber für die Datierung und Kontextualisierung der Proben war er weiterhin von Stein abhängig. Steins Befunde über Herkunft und Alter der spezifischen Proben sowie der anderen, zu Vergleichszwecken konsultierten Papiere waren für Wiesner essentiell. So befand sich etwa unter den Manuskripten von Stein die Papierprobe T.XII.a.ü.Ia, mit deren Hilfe Wiesner seine historische Hypothesen zur Erfindung des Hadernpapiers durch die Chinesen validieren zu können glaubte:

[W] as mir sehr am Herzen liegt, das ist das Papier T XII a ü Ia. Es ist das merkwürdigste Papier, das mir unter den Chinesischen Sachen untergekommen ist. Es liefert den directen Beweis, der bis dahin auf Grund der materiellen Prüfungen noch nicht geführt werden konnte, dass die Chinesen aus Hadern allein Papier erzeugten, während ich früher nur nachweisen konnte, dass sie Hadern als Surrogat der Papiererzeugung benutzten. (Wiesner 1910: LHAS, 10/fols. 115, Hervorhebung im Original)

Als Stein ihm bestätigte, dass T.XII.a.ü.I ${ }^{\mathrm{a}}$ chinesischer Provenienz war, bedeutete dies für Wiesner, dass er damit das erste chinesische Hadernpapier in Händen hielt (Stein 1911: MA8, 3.5.83.A1.18). Damit konnte er in seiner Publikation „Hadernpapiere“ (1911) die Chinesen zu den tatsächlichen Erfindern des reinen Hadernpapiers erklären.

Seinem Kollegen und früheren Kooperationspartner Hoernle waren Wiesners Ausführungen in den „Hadernpapieren“ (1911) zu gewagt. In einem Brief an Wiesner kritisierte Hoernle vor allem zwei Punkte: Erstens 
befand er es als nicht eindeutig bewiesen, dass es sich bei T.XII.a.ü.I ${ }^{a}$ um reines Hadernpapier handelte (Hoernle 1912: MA8, 3.5.83.A1.18). ${ }^{34}$ Ob man überhaupt nachweisen konnte, mikroskopisch oder auf andere Weise, dass die zerstampfte Füllmasse des Papiers wirklich aus reinen Hadern bestand, fragte Hoernle. Damit war Hoernle nicht allein. Wiesner erhielt zwar größtenteils positive Reaktionen, doch gab es unter Historikern auch Skepsis gegenüber seinen naturwissenschaftlichen Methoden. Bereits 1887 hieß es in den „Notizen der Mitteilungen des Instituts für österreichische Geschichtsforschung“ (MIÖG): „W. [Anm.: Wiesner] stellt eine eingehendere Abhandlung in Aussicht, welche auch die für die Untersuchung der alten Papiere wichtige, aber noch ungelöste Frage: „Welches sind die Grenzen der Sicherheit bei der mikroskopischen Untersuchung der Papierfasern?" beantworten wird“ (Mühlbacher 1887: 115).

Zweitens bemängelte Hoernle, dass nur so wenig Material verfügbar war, um Wiesners These zur Brücke zwischen arabischem und chinesischem Papier zu stützen - dass also die Araber die Herstellung reinen Hadernpapiers von den Chinesen erlernt hatten. ${ }^{35}$ Hoernle war „noch nicht recht überzeugt dass dieser Punkt historisch schon entschieden ist" - dieser Brief wurde bereits am Anfang des Aufsatzes zitiert (Hoernle 1912: MA8, 3.5.83.A1.18). Hoernle hielt es zwar für möglich, dass die Chinesen die Erfinder des reinen Hadernpapiers waren. Die wenigen Überreste reinen Hadernpapiers chinesischer Herkunft deuteten für ihn aber eher darauf hin, dass die Chinesen Hadern meistenteils nur als Zusatzstoff verwendet hatten, während die Araber als die Erfinder des reinen Hadernpapiers breiter Anwendung anzusehen waren.

Für Wiesners Status als Experte war diese Fachdiskussion mit Hoernle von hoher Bedeutung: Indem Hoernle Wiesner für seine historischen Hypothesen kritisierte, erkannte Hoernle ihn zugleich als Papierhistoriker an - als Kollegen auf Augenhöhe, dessen papierhistorische Forschung ernst zu nehmen war. Wiesner seinerseits zögerte nicht mehr, eigenständige Positionen zu vertreten und sich inhaltlich von früheren Kooperationspartnern abzugrenzen. 1902 hatte Wiesner noch abgelehnt, sich auf eine „paläographische Kontroverse“ mit Karabacek einzulassen und sich darauf beschränkt, auf naturwissenschaftlich-technischer Basis begründete Annahmen zu formulieren und zu verteidigen. Bereits 1904, endgültig aber 1911 bewegte Wiesner sich wie selbstverständlich auf dem Feld der Papiergeschichte als anerkannte Autorität, auch wenn renommierte Kollegen wie Karabacek und Hoernle ihm inhaltlich widersprachen. Wiesner hatte sich die Forschungsfragen des ihm ursprünglich fremden Feldes zu eigen gemacht, war in die Riege der Historiker aufgestiegen und konnte sich dort behaupten. Stein nannte ihn schließlich sogar den „Bahnbrecher moderner Forschung in der Geschichte des Papiers" (Stein 1910: MA8, 3.5.83.A1.18). 


\section{Offene und geschlossene interdisziplinäre Kooperationen in der Papierforschung um 1900}

Eine umfassende Geschichte der historischen Papierforschung, wie auch der historischen Hilfswissenschaften allgemein, ist noch nicht geschrieben. Unser Aufsatz gibt einen ersten Einblick in die Transformation und Professionalisierung dieses Feldes, vor allem im letzten Drittel des 19. Jahrhunderts. Dabei ist unser Beispiel in mancherlei Hinsicht außergewöhnlich. So war es kein Zufall, dass die fruchtbare Zusammenarbeit von Paläographie und Pflanzenphysiologie gerade in Wien ihren Ausgang nahm. Wie erwähnt, war hier 1854 das IÖG begründet worden, dass sich bewusst auch naturwissenschaftlich-technischen Methoden gegenüber öffnete. Diese spezifischen lokalen Bedingungen begünstigten es erheblich, dass die Paläographie sich um eine Kooperation mit einem Naturwissenschaftler bemühte. Doch war es ein Glücksfall, dass gerade Wiesner angesprochen wurde. Denn Wiesner war kein üblicher Botaniker; er war ein chemisch versierter Pflanzenphysiologe mit umfassenden Kenntnissen in technischer Mikroskopie und Warenkunde, inklusive Verfahren der Papierherstellung. Damit war Wiesner als fachlicher Grenzgänger der ideale Kooperationspartner für die Paläographie. Wie nur wenige in Europa verfügte er über alle Kenntnisse und Fähigkeiten, um die alten Manuskripte zu untersuchen: Wiesner konnte nicht nur die botanische Identität von Pflanzenfasern feststellen, sondern kannte auch zahlreiche weitere Materialeigenschaften von Papier, die sich mikroskopisch und mikrochemisch analysieren ließen.

Damit kommen wir zu der Frage, inwiefern dieser Fall unser Verständnis wissenschaftlicher Kooperationen erweitert - insbesondere unser Verständnis von heterogenen Kooperationen über Disziplinengrenzen hinweg. Es mag überraschen, dass zwei Disziplinen wie Paläographie und Botanik, die sich in Fragestellungen, Methoden und Kategorien deutlich unterscheiden, erfolgreich kooperierten. Die Anforderungen derart interdisziplinärer Kooperation an Akteure sind erheblich; so ist für den Erfolg neben der inhaltlichen Expertise (contributory expertise) auch ein hohes Maß an Integrationsexpertise (interlocking expertise) gefragt. Wie oben ausgeführt, hatte Wiesner bereits zu Beginn der Zusammenarbeit umfassende Sachkenntnis der mikroskopisch-technischen Papieranalyse anzubieten; zudem war er bereit, sich auf die besonderen Herausforderungen historischer Proben einzustellen und mit Paläographen in ein Gespräch über Fragen der Datierung einzutreten. Mit Hilfe seiner Integrationsexpertise verstand es Wiesner, die disziplinären Differenzen zu überbrücken. So erweiterte er die historischen Methoden der Paläographen und beantwortete ihre Fragestellungen nicht nur, sondern richtete eigene Fragen an die Geschichte des Papiers. Dabei kamen ihm die Kooperationspartner mit ihrer Integrati- 
onsexpertise entgegen: Karabacek, Hoernle und Stein - kenntnisreich mit Blick auf die Herkunft und Datierung der Manuskripte - waren bereit, die naturwissenschaftlich erhobenen Daten als Evidenz für historische Interpretationen anzuerkennen und zu nutzen. Durch dieses glückliche Zusammentreffen verlief die Kooperation in allen drei Fällen produktiv, obschon die beiden Arbeitsfelder sich in zentralen Aspekten stark unterschieden.

Im Zuge der beidseitigen Annäherung und Erweiterung der Integrationsexpertise wandelte sich auch die Form und der Charakter der Kooperation. Nicht nur war Wiesner bereit und fähig, seine Expertise mit den Anliegen der Paläographen zu integrieren; er begann zunehmend, die Fragen und Methoden des Feldes auf originelle Weise weiterzuentwickeln und gewann eigenständiges Interesse an dem Gebiet. Im Gegenzug waren die Paläographen zunehmend bereit, das Potential seiner empirischen Befunde anzuerkennen. So antwortete Wiesner anfangs reaktiv auf spezifisch formulierte Forschungsfragen. In einer solchermaßen „geschlossenen Kooperation" arbeitete Wiesner als Experte seinem Kooperationspartner zu, wobei das Erkenntnisziel bereits abgesteckt und festgelegt war. Wiesner war sich zwar über die Bedeutung seiner Beiträge für die Papiergeschichte bewusst, enthielt sich aber vorerst noch einer eigenen Beteiligung an historischen Fragen.

Schon die Zusammenarbeit von Wiesner mit Karabacek, eindeutig dann die Zusammenarbeit mit Hoernle und Stein entwickelte sich jedoch zu einer „offenen Kooperation“. Hoernle wandte sich an Wiesner mit einem ergebnisoffenen Anliegen. Wiesner konnte Forschungsfragen und Methoden selbständig und eigeninitiativ festlegen; so untersuchte er etwa die verschiedene Leimung des Papiers, woran weder Karabacek noch Hoernle von sich aus gedacht hätten. Offene Kooperationen können zu Ergebnissen führen, die für eine, zuweilen auch für beide Seiten unerwartet sind. Im besten Fall arbeiten die Kooperationspartner dabei auf Augenhöhe, akzeptieren beidseitig die sich ergänzenden Kenntnisse und Fähigkeiten und legen ein hohes Maß an Integrationsexpertise an den Tag. Wiesner erweiterte in der Zusammenarbeit seine Sachkenntnisse sogar so weit, dass er begann, als Historiker eigenen Rechts zu agieren - ohne allerdings seine disziplinäre Identität als Pflanzenphysiologie aufzugeben. Er bereicherte die Methoden und Forschungsansätze der Papiergeschichte, erarbeitete aber zudem Befunde über die Eigenschaften und Verarbeitung von Pflanzenfasern, die für sein ursprüngliches Forschungsfeld von Interesse waren.

Eindrücklich führt diese Episode damit vor Augen, dass Kooperationen dynamische Prozesse sind. Sie formen und entwickeln sich unter dem Einfluss von Expertisen, Eigeninitiative, Rezeptionsbereitschaft und Toleranz der Kooperationspartner; entfalten sich aber zudem in einem spezifischen lokalen Kontext. Die Zusammenführung von Wissensbeständen und 
Kompetenz ist die Grundlage interdisziplinärer Kooperationen. Um aber beidseitig Initiative und innovative Prozesse $\mathrm{zu}$ ermöglichen, also offene Kooperationsformen, bedarf es darüber hinaus weitgehender Integrationsexpertise. Wären Wiesner, Karabacek, Hoernle und Stein in dieser Hinsicht weniger erfolgreich gewesen, hätten Paläographen deutlich länger nach Baumwollpapier gesucht.

\section{Genutzte Archivbestände}

- MA8: Stadt- und Landesarchiv, Wien, Nachlass von Julius Wiesner 3.5.83.A1.18.

- ÖNB: Österreichische Nationalbibliothek, Wien, Nachlass von Josef Karabacek.

- LHAS: Akademie der Wissenschaften, Budapest, Nachlass von Marc Aurel Stein.

- Bodleian: Bodleian Library, Oxford, Nachlass von Marc Aurel Stein.

\section{Danksagung}

Wir bedanken uns herzlich bei Elsbeth Bösl, Fabian Krämer und Caterina Schürch für hilfreiche Kommentare zu einer früheren Fassung dieses Aufsatzes.

Funding Open Access funding provided by Projekt DEAL.

Open Access Dieser Artikel wird unter der Creative Commons Namensnennung 4.0 International Lizenz veröffentlicht, welche die Nutzung, Vervielfältigung, Bearbeitung, Verbreitung und Wiedergabe in jeglichem Medium und Format erlaubt, sofern Sie den/die ursprünglichen Autor(en) und die Quelle ordnungsgemäß nennen, einen Link zur Creative Commons Lizenz beifügen und angeben, ob Änderungen vorgenommen wurden.

Die in diesem Artikel enthaltenen Bilder und sonstiges Drittmaterial unterliegen ebenfalls der genannten Creative Commons Lizenz, sofern sich aus der Abbildungslegende nichts anderes ergibt. Sofern das betreffende Material nicht unter der genannten Creative Commons Lizenz steht und die betreffende Handlung nicht nach gesetzlichen Vorschriften erlaubt ist, ist für die oben aufgeführten Weiterverwendungen des Materials die Einwilligung des jeweiligen Rechteinhabers einzuholen.

Weitere Details zur Lizenz entnehmen Sie bitte der Lizenzinformation auf http://creativecom mons.org/licenses/by/4.0/deed.de. 


\section{Anmerkungen}

1 Hoernle 1912: MA8, 3.5.83.A1.18; Hervorhebung im Original.

2 Zur Vorgeschichte der Begegnung von Biologie und Geschichtswissenschaften bei der Erforschung der Vergangenheit siehe auch den Aufsatz „Botany and the Science of History, ca.1800-1900“ von (alphabetisch) Fabian Krämer, Kärin Nickelsen, Dana von Suffrin; derzeit unter Begutachtung für die Zeitschrift Isis.

3 Der Botaniker Julius Sachs (1832-1897) war der erste Wissenschaftler überhaupt, der sich für „Pflanzenphysiologie“ habilitierte (1857, Prag). 1875 veröffentlichte Sachs ein Werk zur Geschichte der Botanik vom 16. Jahrhundert bis 1860 (Sachs 1875), in dem er der Pflanzenphysiologie ein zwanzigseitiges Kapitel widmet. Sein österreichischer Kollege Franz Unger (1800-1870) hatte bereits in seinem Lehrbuch zur Anatomie und Physiologie der Pflanzen (1855) einen historischen Abriss über die Geschichte der Pflanzenphysiologie gegeben. Leider gibt es nur wenig jüngere Forschung zur Geschichte der Pflanzenphysiologie im 19. Jahrhundert (Unger 1855), insbesondere mit Blick auf den deutschen Sprachraum (für Ausnahmen siehe z. B.: de Chadarevian 1996; Liu 2019).

$4 \quad$ Kooperation und Zusammenarbeit werden im Weiteren synonym verwendet.

5 Die Kooperationspartner müssen nicht dasselbe übergeordnete Ziel verfolgen. Das Projekt, für das sie zusammenspannen, kann für die Kooperationspartner aus unterschiedlichen Gründen interessant sein, und sie können auf unterschiedliche Weise von der Zusammenarbeit profitieren. So müssen die Ziele nicht nur epistemischer Natur sein: man kann auch darauf setzen, durch Kooperation mit einer anderen Disziplin den eigenen Status zu erhöhen.

6 Andersen erweitert diesen Begriff, um die Zuschreibung von konvergent und divergent: Während Wissenschaftler mit konvergenter contributory expertise in derselben Disziplin ausgebildet werden, beziehen Wissenschaftler mit divergenter contributory expertise ihre Expertise aus verschiedenen Disziplinen und können ihre Fähigkeiten zu einem neuen Feld zusammenführen, wie beispielsweise in der Geomikrobiologie oder den integrativen Neurowissenschaften.

7 „[F]or a high degree of integration of the various contributions, the required interlocking is quite extensive" (Andersen 2016: 6).

8 Neuere Forschung zeichnet ein differenziertes Bild des historischen Verhältnisses zwischen den Natur- und Geisteswissenschaften und hinterfragt das Trennungsnarrativ der "Zwei Kulturen“. Vgl. dazu etwa das Forum "The Two Cultures Revisited, The Sciences and the Humanities in a Longue Durée Perspective", in History of Humanities, Spring 2018; darin beispielsweise Tkaczyk (2018). Eine Unterscheidung von Natur- und Geisteswissenschaftlern als distinkte Personen ist vor allem mit Blick auf die Frühe Neuzeit hinfällig, siehe etwa Grafton (2018). Eine signifikante personelle, räumliche und epistemologische Trennung vollzog sich erst im 19. Jahrhundert, siehe beispielsweise Krämer (2018); Hamann (2018); und auch danach blieben die Grenzziehungen porös.

9 Man kann Wiesner zur Gruppe der „peripheral scholars“ zählen, die einen naturwissenschaftlichen oder technischen Hintergrund aufwiesen und sich aus disziplinären Interessen heraus in andere Fächer wie Geschichtswissenschaften oder Orientalistik einbrachten, siehe Wokoeck (2009: 79-80). Wiesners Interesse an (historischen) Verfahren der Papierherstellung ergab sich aus seinem breiten Interesse an - und seiner Kompetenz in - Material- und Warenkunde. Andere Beispiele für zeitgenössische peripheral scholars sind etwa der Physiker Eilhard Wiedemann (1852-1928), der um 1900 zur arabischen Wissenschaftsgeschichte publizierte, oder der Chemiker und Zuckertechnologe Edmund Oskar von Lippmann (1857-1940), der zur Geschichte der Alchemie arbeitete. 
10 Der Nachlass von Josef Karabacek findet sich in der Österreichischen Nationalbibliothek, darin sind 33 Briefe von Wiesner an Karabacek aus den Jahren 1887-1915. Im Wiener Stadt- und Landesarchiv (MA 8) befinden sich im Nachlass Wiesners sechs Briefe von Karabacek aus den Jahren 1884-1911.

11 Etwa Wiesner $(1877,1881,1892,1896,1904 a, 1907)$.

12 Karabacek war sowohl 1894/1895 Dekan als auch 1900/1901 Senator der philosophischen Fakultät.

13 Pinto, Mariana, Early Scientific Analysis of Historical Pigments during the long 19th Century, Universität Utrecht, Dissertation (in Vorbereitung).

14. Deutsche Übersetzung der Autorinnen: „Es ist sehr eigenartig, dass derartige Forschungen fast gleichzeitig und ohne gegenseitige Kenntnis der anderen durchgeführt wurden, von Ihnen, in Wien, von den Professoren Girard und Giry in Paris und von mir in Genf. Dass wir alle zum gleichen Ergebnis kommen, würdigt das Mikroskop."

15 Siehe dazu Wiesner (1902: 3), FN 2: „[D]ie Botaniker erledigten die an sie nur gelegentlich gestellten Fragen doch nicht mit der nöthigen wissenschaftlichen Tiefe und kamen über die landläufigen Angaben nicht hinaus.“ Siehe außerdem Wiesner (1887: 190): „Caruel, den wir als Forscher auf vielen botanischen Gebieten so hoch halten, [ist] bei seiner doch wohl nur gelegentlichen Untersuchung der früher genannten Papiere zu irrthümlichen Resultaten gekommen."

16 Das erste archäologische Werk, das einen Anhang mit den Resultaten chemischer Untersuchungen der Ausgrabungsfunde enthielt war Austen Henry Layards Discoveries in the Ruins of Nineveh and Babylon (1853). Auch Heinrich Schliemanns Mycanae (1878) beinhaltete einen solchen Anhang (Caley 1951).

17 Koautorenschaft war im 19. Jahrhundert zwar keine gänzlich unbekannte Erscheinung, aber vergleichsweise selten, vgl. Csiszar (2018).

18 Wiesner untersuchte zum Beispiel Papierproben von den Paläographen Victor Gardthausen, Leopold Delisle oder Cesare Paoli (1840-1902).

19 Wiesner zählt die Namen der Archivare auf: „Pellegrini (Belluno), C. Paoli (Florenz), Davari (Mantua), Capasso (Neapel), Gnoli (Rom), Banchi (Siena), Promis und Cipolla (Turin), Cecchetti und Bertoldi (Venedig)." Die von Wiesner untersuchten Papierproben sind bis heute im IÖG in einer Kiste mit der Aufschrift „Papyrus- und Papierproben“ archiviert (IÖG, Sammlung Papierproben; keine weitere Signatur). Darin befinden sich zahlreiche Briefumschläge mit den Namen der Archive, Archivaren, dem Alter und der Herkunft der Proben, meist in Sickels kleiner, gedrungenen Handschrift. In der Briefkorrespondenz des Sickel-Nachlasses ließ sich allerdings kein Hinweis auf die Papierprobenvermittlung finden. Vgl. hierzu im Detail: Musil-Gutsch (2020).

20 „V.] Sickel hat mich [...] dringlich ersucht, die Papiere aus der Par. Nationalbiblio [...] (von Delile) [sic!] zu untersuchen.", schreibt Wiesner an Karabacek (1887). In diesem kurzen Satz zeigt sich Sickels Vermittlerrolle sowie sein Interesse an Wiesners Forschung.

21 Wiesner (1887: 253): „Sämmtliche orientalische Papiere, welche ich untersuchte, sind mit Stärkekleister geleimt. [...] Die Grenze zwischen Leimung mit Stärkekleister und Thierleim (Leim) fällt in das Ende des XIII. oder in den Anfang des XIV. Jh.s. In der Serie der Papiere aus Siena erlischt die Stärkeleimung mit dem Jahre 1298 und mit diesem Jahre beginnt auch die Leimung mit Thierleim. In der Serie der Papiere aus Venedig fällt die Grenze in das Jahr 1305, in den Turiner in das Jahr 1323." Vgl. hierzu auch MusilGutsch (2020).

22 Wiesner (1887: 227): „Nimmt man zur Prüfung nur sehr wohlerhaltene Papiere, so ergibt sich eine ziemlich klare Beziehung zwischen der Leimung und dem Alter des $\mathrm{Pa}-$ piers. Ich finde nämlich, dass eine ausgiebige, wenn auch nicht gerade starke Leimung den Papieren aus dem Anfange bis zur Mitte des X. Jh.s n. Chr. zukommt, und erst von da ab eine starke Stärkeleimung vorgenommen wurde. [...] Dass aber eine starke Stärkeleimung erst später aufgekommen ist, möchte ich nach meinen Wahrnehmungen als feststehend betrachten. Als Belegstücke für diese meine Meinung führe ich folgende 
Papiere an [...]. Alle diese Papiere sind stark mit Stärkekleister geleimt und geben sehr auffällige Jodreaction."

23 Zahlreiche Briefe in Wiesners Nachlass sowie Rezensionen seiner Arbeit zeugen von dieser Reaktion. Vgl. Mühlbacher (1888: 479): „[D]ie von Wiesner gewonnen Ergebnisse [...] haben die volle Anerkennung und Zustimmung in Fachkreisen gefunden."

24 Wattenbach (1887: MA8), 3.5.83.A1.18: „Das Baumwollenpapier muß nun ja [...] aufgegeben werden, und die ganze Entwicklung dieser [...] ist in erfreulichster Weise aufgeklärt. Daß ich überall als Repräsentant von Irrthümern erscheine, welche ich nur anderen nachschrieb, weil es damals nicht anders möglich war, muß ich mir ja gefallen laßen."

25 Die „Ausserachtlassung dieses Theiles der Papierprüfung ist die Hauptursache der unsicheren und fehlerhaften Resultate, zu denen viele Beobachter gelangten“ (Wiesner 1887: 196).

26 Die heutige Bezeichnung ist Tetramminkupfersulfat.

27 Siehe auch Wiesner (1902: 18): „Am 19. Februar 1900 richtete Herr Prof. Hoernle an mich das Ersuchen, die materielle Untersuchung der im Besitze der englischen Regierung befindlichen alten ostturkestanischen Papiere zu übernehmen." Briefe zwischen Wiesner und Hoernle finden sich in folgenden Archiven: Letters to A.F.R. Hoernle, 1901-1902, 458, fols. 35-38, 66-71, 76-90, 96-106., Bodleian Library, Oxford, vgl. Diamond, Elizabeth und T.D. Rogers, „Bodleian Library Oxford: Catalogue of the Papers of Sir (Marc) Aurel Stein (1862-1943)“, URL: https://www.britishmuseum.org/pdf/ 16_Diamond-Rogers-Fletcher.pdf; zuletzt aufgerufen am 04.01.2017, 19.

28 In Sanskrit im Brahmi-Alphabet verfasst, aus dem 5. Jh. n. Chr. stammend, war sie eine der ältesten Abhandlungen zur indischen Medizin und obendrein eine bedeutende Entdeckung für die Linguistik. Hoernle veröffentlichte 1897 eine vollständige Edition der Manuskripte, die ihn zum Experten für Sanskritmedizin und alte Medizingeschichte machten.

29 Vgl. den hier zitierten Brief Hoernles an Wiesner vom 10. April 1900.

30 In einer Rezension über Wiesners papierhistorische Arbeiten heißt es: „Diese wichtigen Erkenntnisse auf dem Gebiet der Papierfabrikation waren theilweise nur dadurch mit voller Bestimmtheit zu erlangen, dass die Untersuchungsmethoden weiter entwickelt wurden; so sind sie die Ursache weiterer Ausbildung der Methoden der mikroskopischen Untersuchung des Papiers geworden." Vgl. Kick (1888: 73).

31 Siehe Wiesner (1902: Bodleian, Ms Stein 458, fols. 88): Hier erfährt man, dass Wiesner durch Hoernles „Bemerkungen über die muthmasslich von den Chinesen Ostturkestans angebliche Erfindung des Hadernpapiers [... ] in einigen diesbezüglichen Auffassungen" bestärkt wurde.

32 Ein Brief Wiesners an Stein kündet von der ersten Begegnung zwischen den beiden: „Es war mir eine große Freude, Sie, hochgeehrter Herr, persönlich kennen gelernt zu haben, nachdem mir Ihr Name durch Hoernle und andere hochangesehene Gelehrte und durch die Literatur so rühmlich bekannt wurde und so würde es mich freuen, mit Ihnen in regen wissenschaftlichen Verkehr treten zu können." Wiesner (1902: Ms Stein 112, fols. 35)

33 Für die Zeit von Oktober 1910 bis März 1911 ist die Korrespondenz fast vollständig erhalten. Für den Zeitraum Ende März bis Mitte Mai 1911 haben wir nur noch die Briefe von Stein. Diese geben allerdings Aufschluss über die Datierung der verloren gegangenen Wiesner-Briefe, da sich Stein stets für den vorhergegangenen Brief Wiesners bedankt und dabei das Absendedatum des erhaltenen Briefes nannte. So kann man den Briefwechsel rekonstruieren: nicht erhaltene Briefe von Wiesner vom 21./22. März (erhaltene Antwort Stein: 01.04.1911); nicht erhalten Wiesner: 07.04.1911 (erhaltene Antwort Stein: 11.04.1911); nicht erhalten Wiesner 16.04.1911 (erhaltene Antwort Stein: 19.04.1911); nicht erhaltene Antwort Wiesner: 17.5. (erhaltene Antwort Stein 21.5.).

34 Hoernle 1912: MA8, 3.5.83.A1.18. Auf dem Brief findet sich die Datierung „1. Januar 1911“, doch ist anzunehmen, dass Hoernle der allseits bekannte Fehler zu Jahresbeginn 
unterlief, den Brief noch auf das vorige Jahr zu datieren. Erstens wird im Brief auf die bereits im Juni 1911 erschienene Publikation Wiesners Bezug genommen, zweitens findet sich am Briefende eine handschriftliche Notiz Wiesners, die das Datum angibt, an dem der Brief beantwortet wurde: 15.01.1912.

35 Wiesner (1911: 26): „Da die Chinesen lange vor den Arabern vollständige Hadernpapiere erzeugten und erwiesenermaßen noch in der Zeit, in welcher die arabische Papierbereitung begann, chinesische Papiere mit Hadernzusatz verfertigt wurden, ferner, wie allgemein bekannt, die Chinesen die Araber in der Papierbereitung unterrichteten, so ist wohl nicht mehr zu bezweifeln, daß die Araber von den Chinesen nicht nur die Methode erlernten, ein gefilztes Papier herzustellen, sondern auch in der Anwendung von Hadern zur Papiererzeugung unterrichtet wurden."

\section{Literatur}

Ali-de-Unzaga, Miriam 2012. „Welch’ herrlicher Anfang!!“ Josef Karabacek and Egyptian Textiles in Fin-de-Siècle Vienna. In: Bernhard Palme und Angelika Zdiarsky (Hg.). Gewebte Geschichte. Stoffe und Papyri aus dem spätantiken Ägypten. Wien: Phoibos: 75-86.

Andersen, Hanne 2016. Collaboration, Interdisciplinarity, and the Epistemology of Contemporary Science. Studies in History and Philosophy of Science (Part A 56): 1-10.

Andersen, Hanne und Susann Wagenknecht 2013. Epistemic Dependence in Interdisciplinary Groups. Synthese (190/11): 1881-1898.

Andrae, Walter 1923. Farbige Keramik aus Assur und ihre Vorstufen in altassyrischen Wandmalereien. Nach Aquarellen von Mitgliedern der Assur-Expedition und nach photographischen Aufnahmen von Originalen im Auftrag der Deutschen Orient-Gesellschaft. Berlin: Scarabaeus-Verlag.

Babler, O. F. 29. Juni 1935. Professor Julius Wiesner. Notes and Queries (CLXVIII): 463.

Bär, Jürgen 2003. Walter Andrae: Ein Wegbereiter der modernen Archäologie. Grabungstechnik, Dokumentation, naturwissenschaftliche Analysen und Alltag. In: Joachim Marzahn (Hg.). Wiedererstehendes Assur. 100 Jahre deutsche Ausgrabungen in Assyrien. Mainz: Zabern: 45-52.

Becker, C. 1920. Joseph von Karabacek. Der Islam (10): 233-238.

Bihl, Wolfdieter 2009. Orientalistik an der Universität Wien: Forschungen zwischen Maghreb und Ost- und Südasien - die Professoren und Dozenten. Wien: Böhlau.

Bod, Rens und Julia Kursell 2015. Introduction: The Humanities and the Sciences. Isis (106/2): 337-340.

Bol, Marjolijn 2017. Technique and the Art of Immortality, 1800-1900. History of Humanities (2/1): 179-199.

Bösl, Elsbeth 2017. Doing Ancient DNA: Zur Wissenschaftsgeschichte der aDNA-Forschung. Bielefeld: Transcript.

Bouterse, Jeroen und Bart Karstens 2015. A Diversity of Divisions: Tracing the History of the Demarcation between the Sciences and the Humanities. Isis (106/2): 341-352.

Bracchi, Eva 2014. Friedrich Rathgen, Pionier der modernen archäologischen Restaurierung. Berliner Beiträge zur Archäometrie (22): 5-13.

Briquet, Charles Moïse 1884. La légende paléographique du papier du coton. Genf: Imprimerie Charles Suchardt.

Brittner, Carl 1943. Friedrich Rathgen. Berliner Museen (64/1): 19-20.

Caldararo, Niccolo Leo 1987. An Outline History of Conservation in Archaeology and Anthropology as Presented through Its Publications. Journal of the American Institute for Conservation (26/2): 85-104.

Caley, Earle R 1951. Early History and Literature of Archaeological Chemistry. Journal of Chemical Education (28/2): 64-66.

Caley, Earle R. 1967. The Early History of Chemistry in the Service of Archaeology. Journal of Chemical Education (44/3): 120-123. 
Capanelli, Emilio und E. Insabato 2000. Guida agli archivi delle personalità della cultura in Toscana tra '800 e '900: L'area pisana. Florenz: Olschki.

Capannelli, Emilio und Marco Lenzi o. J. Clemente Lupi. Archivi di personalità: Censimento dei fondi toscani tra '800 e'900. URL: http://siusa.archivi.beniculturali.it/cgi-bin/ pagina.pl? TipoPag=prodpersona $\&$ Chiave $=458 \&$ RicProgetto=personalita, (5.10.2018).

Chadarevian, Soraya de 1996. Laboratory Science versus Country-House Experiments: The Controversy between Julius Sachs and Charles Darwin. British Journal for the History of Science (29): 17-41.

Clavir, Miriam 1998. The Social and Historic Construction of Professional Values in Conservation. Studies in Conservation (43/1): 1-8.

Corfield, Michael 1988: Towards a conservation profession. In: Victoria Todd (Hg.). Conservation Today: Papers presented at the UKIC 30th Anniversary Conference 1988. London: United Kingdom Institute of Conservation: 4-7.

Csiszar, Alex 2018. The Scientific Journal: Authorship and the Politics of Knowledge in the Nineteenth Century. Chicago: University of Chicago Press.

Daston, Lorraine 2007. Objektivität. 1. Aufl. Frankfurt am Main: Suhrkamp.

Daston, Lorraine und Kathrin Park 2002. Wunder und die Ordnung der Natur. 1150-1750. Berlin: Eichborn.

Davidson, Cathy und Danica Savonick 2017. Digital Humanities: The Role of Interdisciplinary Humanities in the Information Age. In: Julie Klein Thompson, Robert Frodeman und Roberto Carlos dos Santos Pacheco (Hg.). The Oxford Handbook of Interdisciplinarity. 2. Aufl. Oxford: Oxford University Press: 159-172.

Demeulenaere-Douyère, Christiane und Martine François. Girard, Alfred Claude Aimé. Francais savantes. URL: http://cths.fr/an/prosopo.php?id=876 (17.06.2019).

Dupré, Sven 2017. Materials and Techniques between the Humanities and Science: Introduction. History of Humanities (2/1): 173-178.

Erben, Wilhelm 1926. Theodor von Sickel. Denkwürdigkeiten aus der Werdezeit eines deutschen Geschichtsforschers. München: Oldenbourg.

Gabriel, Mordecai L. 2007. Wiesner, Julius Von. In: Michael Berenbaum und Fred Skolnik (Hg.). Encyclopaedia Judaica. 2. Aufl. Detroit: Macmillan Reference USA: 55-56.

Galison, Peter 2003. The Collective Author. In: Mario Biagioli und Peter Galison (Hg.). Scientific Authorship. Credit and Intellectual Property in Science. New York: Routledge: 325-358.

Gannett, Lisa 2019. The Human Genome Project. In: Edward N. Zalta (Hg.). The Stanford Enyclopedia of Philosophy (Winter 2019 Edition). URL 〈https://plato.stanford.edu/ archives/win2019/entries/human-genome//.

Gilberg, Mark 1987. Friedrich Rathgen: The Father of Modern Archaeological Conservation. Journal of the American Institute for Conservation (26/2): 105-120.

Giry, Arthur o. J. French Historian. Encyclopedia Britannica. https://www.britannica.com/ biography/Arthur-Giry (9.11.2019).

Gloy, Karen 2002. „Kultur“ versus naturwissenschaftlich-technologische Welt. Ein Tableau. In: Karen Gloy (Hg.). Im Spannungsfeld zweier Kulturen. Eine Auseinandersetzung zwischen Geistes- und Naturwissenschaft, Kunst und Technik. Würzburg: Königshausen und Neumann: 9-30.

Gottschalk, Hans 1977. Joseph Ritter v. Karabacek. In: Fritz Wagner (Hg.). Neue Deutsche Biographie. Berlin: Duncker und Humbolt: 140.

Grafton, Anthony 2018. Philological and Artisanal Knowledge Making in Renaissance Natural History: A Study in Cultures of Knowledge. History of Humanities (3/1): 39-55.

Grierson, George A. 1919. Augustus Frederic Rudolf Hoernle. Journal of the Royal Asiatic Society of Great Britain E Ireland (51/1): 114-124.

Guerra, M. F. 2008: Archaeometry and Museums: Fifty Years of Curiosity and Wonder. Archaeometry (50/6, 1): 951-967.

Hamann, Julian 2018. Boundary Work between Two Cultures: Demarcating the Modern Geisteswissenschaften. History of Humanities (3/1): 27-38.

Härtel, Reinhard 2002. Geschichte des Mittelalters und Historische Hilfswissenschaften. In: Karl Acham (Hg.). Geschichte der österreichischen Humanwissenschaften. 1. Aufl. Wien: Passagen-Verlag: 127-160. 
Hauser, Stefan 2005. Quellen - Material. Historiker, Archäologen und das Schweigen der Steine. In: Konrad Hitzl (Hg.). Methodische Perspektiven in der klassischen Archäologie. Tübingen: Deutscher Archäologen-Verband.

Henning, Eckart 2000: Die Historischen Hilfswissenschaften - historisch gesehen! In: Friedrich Beck (Hg.). Vom Nutz und Frommen der historischen Hilfswissenschaften. Neustadt an der Aisch: Degener: 11-22.

Hiecke, R. 1942. Friedrich Rathgen zum Gedächtnis. Deutsche Kunst und Denkmalpflege (5/6, 43): 91.

Jardine, Lisa 2010: C.P. Snow's Two Cultures Revisited. Christ's College Magazine (235): 49-57.

Jones, Elizabeth 2018. Ancient DNA: A History of the Science Before Jurassic Park. Studies in History and Philosophy of Science Part C: Studies in History and Philosophy of Biological and Biomedical Sciences (68): 1-14.

Jost, Halfmann und Johannes Rohbeck 2007. Zur Kulturdifferenz zwischen den Wissenschaften. In: Jost Halfmann und Johannes Rohbeck (Hg.). Zwei Kulturen der Wissenschaft - revisited. Göttingen: Velbrück Wissenschaft: 7-15.

Karabacek, Josef 1883. Die Theodor Graf'schen Funde in Aegypten. Der Papyrusfund und ElFaijûm, die textilen Gräberfunde. Wien: Gerold.

Karabacek, Josef 1885. Papyrus Erzherzog Rainer. Monatsschrift für den Orient (8): 160-165.

Karabacek, Josef 1887. Das arabische Papier. Mittheilungen aus der Sammlung der Papyrus Erzherzog Rainer 2-3: 87-178.

Kick, Friedrich 1888. Die mikroskopische Untersuchung des Papiers (Rezension). Technische Blätter (20): 72-73.

Kisser, Josef 1963. Julius Ritter von Wiesner. In: Hugo Freund (Hg.). Geschichte der Mikroskopie: Leben und Werk großer Forscher, Bd. 1: Biologie. Frankfurt am Main: Umschau: 347-356.

Koch, Walter 2005. Quellen „in die Hand genommen“: Die historischen Hilfswissenschaften und ihr Beitrag für geisteswissenschaftliche Großprojekte. Akademie Aktuell (2): 12-19.

Krämer, Fabian 2018. Shifting Demarcations: An Introduction. History of Humanities (3/1): $5-14$.

Lessing, Julius 1900. Das halbe Jahrhundert der Weltausstellungen: Vortrag gehalten in der Volkswirthschaftlichen Gesellschaft zu Berlin März 1900. Berlin: Simion.

Lhotsky, Alphons 1954. Geschichte des Instituts für österreichische Geschichtsforschung 1854-1954: Festgabe zur Hundert-Jahr-Feier des Instituts. Graz: Böhlau.

Linsbauer, Karl 1917. Julius Wiesner. Mitteilungen des Naturwissenschaftlichen Vereins für Steiermark (53): 1-13.

Liu, Daniel 2019. The Artificial Cell, the Semipermeable Membrane, and the Life That Never Was, 1864-1901. Historical Studies in the Natural Sciences, (49/5): 504-555. https:// doi.org/10.1525/hsns.2019.49.5.504

Lökkos, Antal o. J. Briquet, Charles-Moïse. Historisches Lexikon der Schweiz. URL: http:// www.hls-dhs-dss.ch/textes/d/D31460.php?topdf=1 (5.10.2018).

Löwenfeld, Samuel 1889. Wilhelm Wattenbach. Preußische Jahrbücher (64): 408-429.

Lupi, Clemente 1875. Manuale Di Paleografia Delle Carte. Florenz: Nabu Press.

Marchand, Suzanne L. (1994) The Rhetoric of Artifacts and the Decline of Classical Humanism: The Case of Josef Strzygowski. History and Theory (33/4): 106.

Mauthe, Gabriele 1999. Josef Ritter von Karabacek. In: Gabriele Mauthe und Christian Gastgeber (Hg.). Die Direktion der Hofbibliothek zur Jahrhundertwende Josef Ritter von Karabacek, Direktor der K.K. Hofbibliothek in Wien (1899-1917): Katalog zur Ausstellung im Papyrusmuseum. Wien: Österreichische Nationalbibliothek: 11-42.

Mehr, Christian 2009. Kultur als Naturgeschichte: Opposition oder Komplementarität zur politischen Geschichtsschreibung 1850-1890? Berlin: De Gruyter.

Molisch, Hans 1916. Julius von Wiesner (Nachruf). Berichte der deutschen Botanischen Gesellschaft (34/11): 71-99.

Mommsen, Wolfgang 1997: Kultur und Wissenschaft im kulturellen System des Wilhelminismus. Die Entzauberung der Welt durch Wissenschaft und ihre Verzauberung durch Kunst und Literatur. In: Rüdiger vom Bruch (Hg.). Kultur und Kulturwissenschaften um 1900 II: Idealismus und Positivismus. Stuttgart: Franz Steiner: 24-40. 
Mühlbacher, Engelbert 1887. Notizen. Mitteilungen des Instituts für Österreichische Geschichtsforschung (8): 114-115.

Mühlbacher, Engelbert 1888. Julius Wiesner, Die mikroskopische Untersuchung des Papiers mit besonderer Berücksichtigung der ältesten orientalischen und europäischen Papiere (Rezension). Mitteilungen des Instituts für Österreichische Geschichtsforschung (9/9): $477-485$

Mühlberger, Kurt 2009. Das „Antlitz“ der Wiener Philosophischen Fakultät in der zweiten Hälfte des 19. Jahrhunderts: Struktur und personelle Erneuerung. In: Johannes Seidl (Hg.). Eduard Suess und die Entwicklung der Erdwissenschaften zwischen Biedermeier und Sezession. Göttingen: V\&R Unipress: 67-104.

Musil-Gutsch, Josephine 2020. On the Same Page: Investigating Material Remains of the Knowledge-Producing Practices in Sciences and Humanities. History of Humanities, Spring Issue 2020.

Needham, Joseph and Tsien Tsuen-Hsuin 1985. Science and Civilisation in China. Volume 5. Chemistry and Chemical Technology. Part 1. Paper and Printing. Cambridge: Cambridge University Press.

Nickelsen, Kärin 2014. Kooperation und Konkurrenz in den Naturwissenschaften. In: Ralph Jessen (Hg.). Konkurrenz in der Geschichte. Frankfurt am Main: Campus: 353-379.

Nickelsen, Kärin. Schürch, Caterina 2020. Zur Dynamik disziplinenübergreifender Forschungsfelder. In: Michael Jungert, Andreas Frewer und Erasmus Mayr (Hg.). Wissenschaftsreflexion. Interdisziplinäre Grundlagen und ethische Perspektiven. Paderborn: Mentis.

o. A. 2014. Introduction. The American Historical Review (119/5): 1492-1499.

o. A. und Engelbert Mühlbacher 1887. Notizen. Mitteilungen des Instituts für Österreichische Geschichtsforschung (30/3): 114-141.

Pagliai, L. 1919. Necrologia: Clemente Lupi. Archivio storico italiano (77/1): 199-216.

Parker, John N., Bart Penders und Niki Vermeulen 2013. Understanding Life Together: A Brief History of Collaboration in Biology. Endeavour (37/3): 162-171.

Pinto, Mariana 2020 (in Vorbereitung). Early Scientific Analysis of Historical Pigments during the long 19th Century. Universität Utrecht: Dissertation.

Plenderleith, Harold J. 1998. A History of Conservation. Studies in Conservation (43/3): $129-43$.

Rathgen, Friedrich 1903. Konservierung babylonischer Tontafeln. Chemiker-Zeitung (27/66): 811.

Rathgen, Friedrich 1908. Über Konservierung babylonischer Tontafeln. Museumskunde (IV): 12-24, 88-91.

Rathgen, Friedrich 1913. Über Ton und Glas in alter und uralter Zeit. Chemiker-Zeitung (43): 441.

Real, Leslie 2012. Collaboration in the Sciences and the Humanities: A Comparative Phenomenology. Arts and Humanities in Higher Education (11/3): 250-261.

Redlich, Oswald 1927. Theodor Sickel, Werdezeit und Persönlichkeit. Mitteilungen des Instituts für österreichische Geschichtsforschung (42): 154-164.

Rhodokanakis, Nikolaus 1919. Josef v. Karabacek. In: Akademie der Wissenschaften in Wien (Hg.). Almanach / Akademie d. Wissenschaften in Wien für das Jahr 1919. Wien: Österreichische Akademie der Wissenschaften: 188-198.

Riederer, Josef 1976. Denkschrift zur Eröffnung des Rathgen-Forschungslabors der Staatlichen Museen Preußischer Kulturbesitz. Berlin: Rathgen-Forschungslabor.

Rodenberg, Carl 1898. Wattenbach, Wilhelm. Allgemeine Deutsche Biographie (44): 439-443.

Ruske, Walter 1971. 100 Jahre Materialprüfung in Berlin: Ein Beitrag zur Technikgeschichte. Berlin: Bundesamt für Materialprüfung.

Sachs, Julius 1875. Geschichte der Botanik vom 16. Jahrhundert bis 1860. München: Oldenburg.

Schleier, Hans 2003. Geschichte der deutschen Kulturgeschichtsschreibung. Waltrop: Spenner.

Selander, Anna 2008. The Egypt Connection: Theodor Graf und Josef Karabacek. In: Johanna Holaubek, Hana Navrátilová, und Wolf B. Oerter (Hg.) Ägypten und Österreich IV. Begegnungen. Prag: Czech Institute of Egyptology: 83-100. 
Sickel, Theodor 1872. Literaturbericht zu Wattenbach, Wilhelm: Schriftwesen im Mittelalter, 1871. Historische Zeitschrift (27): 446.

Sims-William, Ursula 2012. Rudolf Hoernle and Sir Aurel Stein. In: The British Museum. Sir Aurel Stein: Colleagues and Collections. https://www.britishmuseum.org/research/ publications/research_publications_series/2012/sir_aurel_stein.aspx (28.3.2019).

Tkaczyk, Viktoria 2018. Whose Larynx Is It? Fields of Scholarly Competence around 1900. History of Humanities (3/ 1): 57-73.

Troelenberg, Eva-Maria 2011. Eine Ausstellung wird besichtigt. Die Münchner „Ausstellung von Meisterwerken muhammedanischer Kunst" 1910 in kultur- und wissenschaftsgeschichtlicher Perspektive. Frankfurt am Main: Lang.

Unger, Jakob und L. Debbert 1988. Vor 100 Jahren: Gründung des ersten Museumslabors der Welt. Neue Museumskunde (31/2): 132-135.

Unger, Franz 1855. Anatomie und Physiologie der Pflanzen. Leipzig: Hartleben.

Wattenbach, Wilhelm 1875. Das Schriftwesen im Mittelalter. 2. Auflage. Leipzig: S. Hirzel.

Wattenbach, Wilhelm 1888. Nachrichten. Neues Archiv der Gesellschaft für Ältere Deutsche Geschichtskunde zur Beförderung einer Gesamtausgabe (13): 650-669.

Welsh, Caroline und Stefan Willer 2008. Einleitung: Die wechselseitige Bedingtheit der Wissenskulturen - ein Gegenentwurf zur Trennungsgeschichte. In: Caroline Welsh und Stefan Willer (Hg.). „Interesse für bedingtes Wissen“: Wechselbeziehungen zwischen den Wissenskulturen. München: Fink: 9-20.

Wiesner, Julius 1877. Die Entstehung des Chlorophylls in der Pflanze. Eine physiologische Untersuchung. Wien: Hölder.

Wiesner, Julius 1881. Elemente der wissenschaftlichen Botanik. Wien: Hölder.

Wiesner, Julius 1887. Die Faijûmer und Uschmûner Papiere. Eine naturwissenschaftliche, mit Rücksicht auf die Erkennung alter und moderner Papiere und auf die Entwicklung der Papierbereitung durchgeführte Untersuchung. Mittheilungen aus der Sammlung der Papyrus Erzherzog Rainer (2-3): 179-260.

Wiesner, Julius 1892. Die Elementarstructur und das Wachsthum der lebenden Substanz. Wien: Hölder.

Wiesner, Julius 1896. Untersuchungen über das photochemische Klima von Wien, Cairo und Buitenzorg (Java). Wien: Hof- und Staatsdruckerei.

Wiesner, Julius 1902. Mikroskopische Untersuchung alter ostturkestanischer und anderer asiatischer Papiere nebst histologischen Beiträgen zur mikroskopischen Papieruntersuchung. Wien: Hof- und Staatsdruckerei.

Wiesner, Julius 1904a. Das Pflanzenleben des Meeres. Wien: W. Braumüller.

Wiesner, Julius 1904b. Ein neuer Beitrag zur Geschichte des Papieres (vorgelegt in der Sitzung am 9. Dezember 1903) (=Sitzungsberichte der Kais. Akademie der Wissenschaften in Wien, Philosophisch-historische Klasse (CXLVIII/6)). Wien: Gerold in Komm.

Wiesner, Julius 1907. Der Lichtgenuss der Pflanzen. Photometrische und physiologische Untersuchungen mit besonderer Rücksichtnahme auf Lebensweise, geographische Verbreitung und Kultur der Pflanzen. Leipzig: Engelmann.

Wiesner, Julius 1910. Die Beziehungen der Pflanzenphysiologie zu den anderen Wissenschaften: Inaugurationsrede, gehalten am 24. October 1898 im Festsaale der Universität. In: Julius Wiesner (Hg.). Natur-Geist-Technik: Ausgewählte Reden, Vorträge und Essays. Leipzig: Engelmann: 101-138.

Wiesner, Julius 1911. Über die ältesten bis jetzt aufgefundenen Hadernpapiere. Ein neuer Beitrag zur Geschichte des Papiers. Wien: Hölder.

Wilhelm, Friedrich 1972. Hoernle, Rudolf. Neue Deutsche Biographie (9): 361-362.

Wininger, Salomon 1979. Wiesner, Julius. In: Große jüdische Nationalbiographie. Nendeln, Liechtenstein: Kraus: 282-283.

Wokoeck, Ursula 2009. German Orientalism. The Study of the Middle East and Islam from 1800 to 1945 . New York: Routledge.

Wray, K. Brad 2006. Scientific Authorship in the Age of Collaborative Research. Studies in History and Philosophy of Science (Part A 37/3): 505-514.

Wray, K. Brad 2002. The Epistemic Significance of Collaborative Research. Philosophy of Science (69): 150-168.

Wurzbach, Constantin von 1888. Wiesner, Julius. In: Biographisches Lexikon des Kaiserthums Oesterreich, Bd. 56. Wien: Zamarski: 88-92. 
Zeumer, Karl 1889. Wilhelm Wattenbach. Historische Zeitschrift (80): 75-85.

Hinweis des Verlags Der Verlag bleibt in Hinblick auf geografische Zuordnungen und Gebietsbezeichnungen in veröffentlichten Karten und Institutsadressen neutral.

Josephine Musil-Gutsch

Historisches Seminar, Abt. Wissenschaftsgeschichte

LMU München

Geschwister-Scholl-Platz 1

80539 München

Deutschland

j.musilgutsch@campus.lmu.de

Kärin Nickelsen

Historisches Seminar, Abt. Wissenschaftsgeschichte

LMU München

Geschwister-Scholl-Platz 1

80539 München

Deutschland

K.Nickelsen@lmu.de 